NUREG/CR-6149

SAND93-2478

\title{
Applications of Fiber Optics in Physical Protection
}

Manuscript Completed: November 1993

Date Published: March 1994

Prepared by

T. H. Buckle

Sandia National Laboratories

Albuquerque, NM 87185-0781

Prepared for

Division of Fuel Cycle Safety and Safeguards

Office of Nuclear Material Safety and Safeguards

U.S. Nuclear Regulatory Commission

Washington, DC 20555-0001

NRC FIN L1387 
\begin{tabular}{lllll}
$\ldots$ & $\ddots$ & - & $\ldots$ \\
\hline
\end{tabular} 


\section{DISCLAIMER}

This report was prepared as an account of work sponsored by an agency of the United States Government. Neither the United States Government nor any agency thereof, nor any of their employees, make any warranty, express or implied, or assumes any legal liability or responsibility for the accuracy, completeness, or usefulness of any information, apparatus, product, or process disclosed, or represents that its use would not infringe privately owned rights. Reference herein to any specific commercial product, process, or service by trade name, trademark, manufacturer, or otherwise does not necessarily constitute or imply its endorsement, recommendation, or favoring by the United States Government or any agency thereof. The views and opinions of authors expressed herein do not necessarily state or reflect those of the United States Government or any agency thereof. 


\section{DISCLAIMER}

Portions of this document may be illegible in electronic image products. Images are produced from the best available original document. 


\begin{abstract}
The purpose of this NUREG is to provide technical information useful for the development of fiber-optic communications and intrusion detection subsystems relevant to physical protection. There are major sections on fiber-optic technology and applications. Other topics include fiber-optic system components and systems engineering. This document also contains a glossary, a list of standards and specifications, and a list of fiber-optic equipment vendors.
\end{abstract}




\section{Contents}

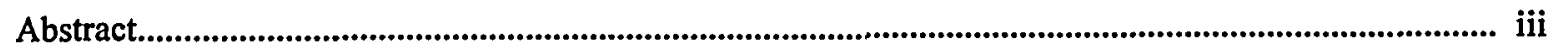

Acknowledgments........................................................................................................................................... ix

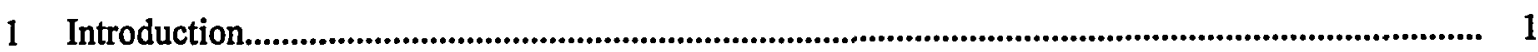

1.1 Discussion of NRC Requirements........................................................................................... 1

1.2 Purpose of Document......................................................................................................... 1

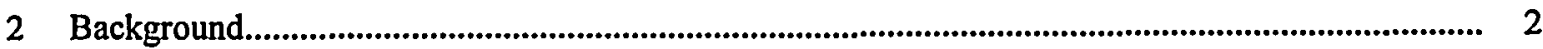

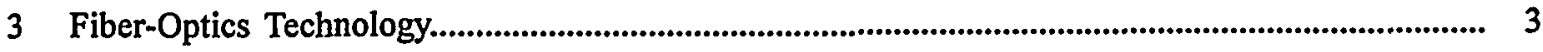

3.1 Theory of Operation............................................................................................................ 3

3.1.1 Optical Fiber Transmission Fundamentals.................................................................. 3

3.1.2 Sensing Phenomena........................................................................................... 4

3.2 System Components............................................................................................................. 5

3.2.1 Optical Fiber and Cable............................................................................................... 5

3.2.2 Connectors and Splices.................................................................................................. 7

3.2.3 Emitters (Light Sources)................................................................................................. 10

3.2.4 Detectors (Light Receivers)............................................................................................. 10

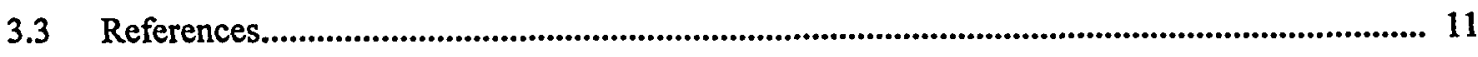

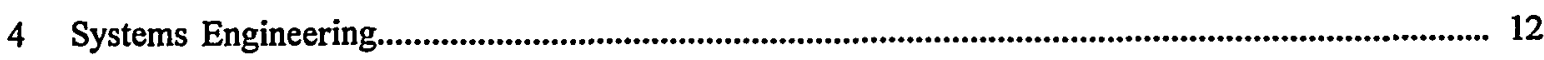

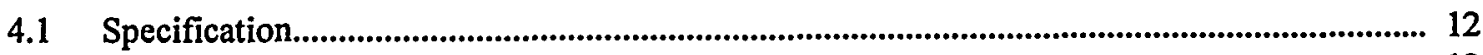

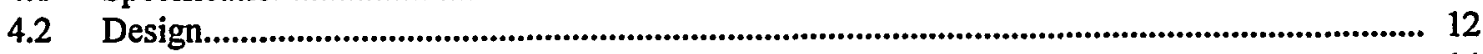

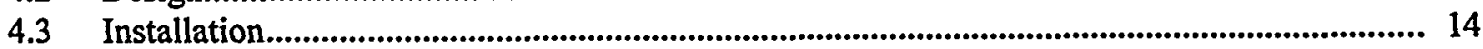

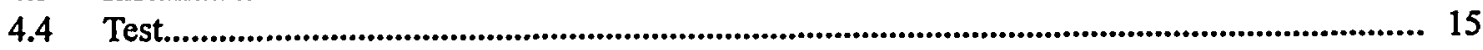

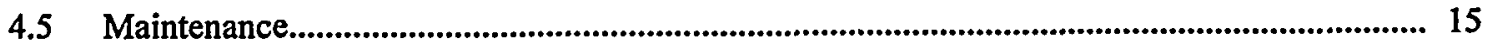

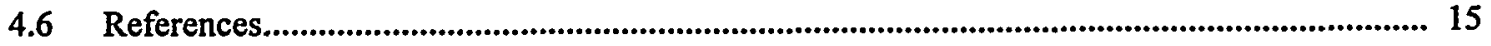

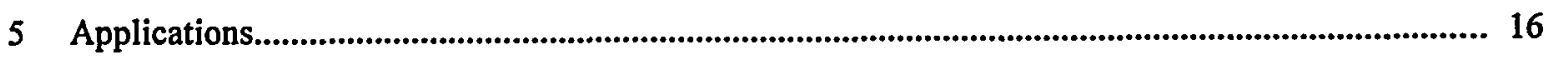

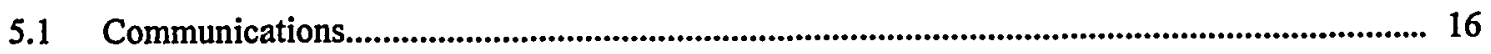

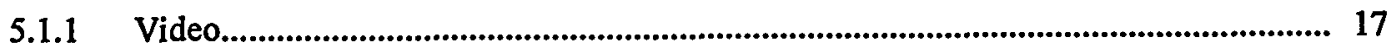

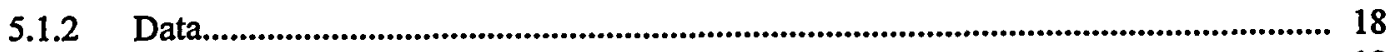

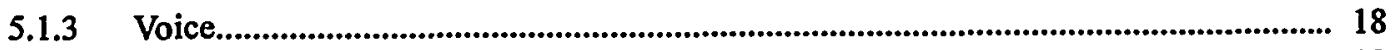

5.1.4 Communications Products.......................................................................................... 19

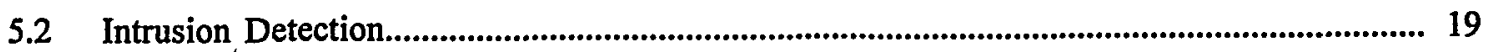

5.2.1 Light Disturbance Products........................................................................................ 19

5.2.2 Optical Continuity Products............................................................................................ 22 
Contents

5.3 Tamper-Indicating Devices (Loop Systems)............................................................................. 22

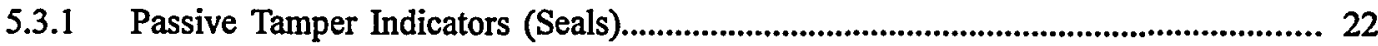

5.3.2 Active Tamper Indicators........................................................................................ 23

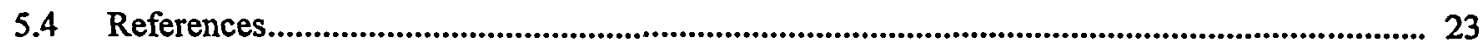

Appendix A, Optical Fiber Theory of Operation............................................................................. 25

A.1 Refraction, Total Internal Reflection, and Numerical Aperture.............................................. 25

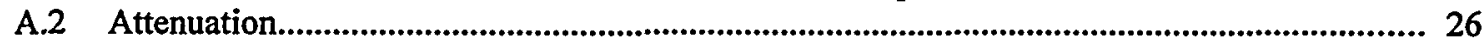

A.3 Dispersion Limitations of Optical Fibers.............................................................................. 27

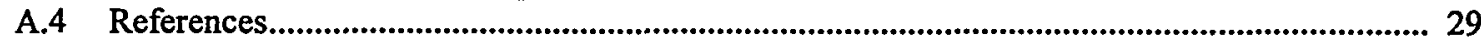

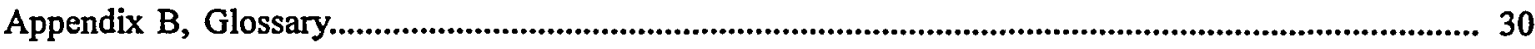

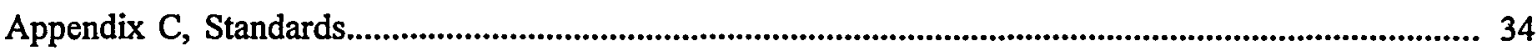

Appendix D, Partial Listing of Fiber-Optics Equipment Suppliers.......................................................... 35

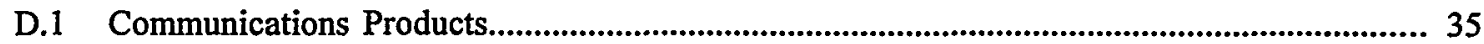

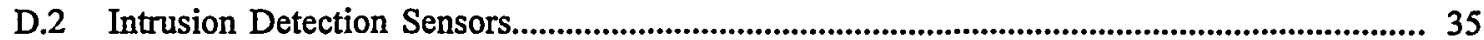

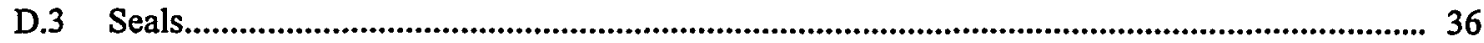

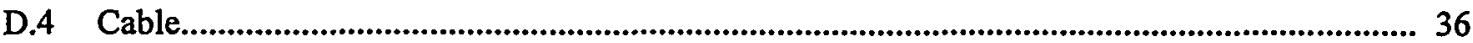

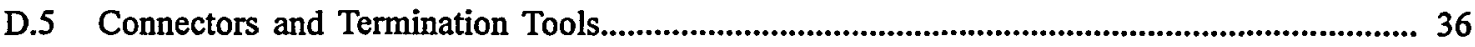

D.6 Splices, Splicing Equipment, and Closure Systems........................................................................ 37

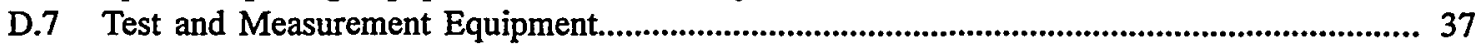




\section{Figures}

3-1 Core and cladding of optical fiber...................................................................................................... 3

3-2 Optical fiber guides light................................................................................................................ 3

3-3 Step-index multimode fiber................................................................................................................ 4

3-4 Graded-index multimode fiber............................................................................................................. 4

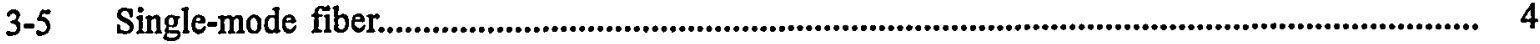

3-6 Effects of microbending on fiber................................................................................................ 5

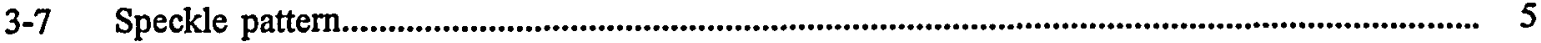

3-8 A basic fiber-optic system..................................................................................................... 5

3-9 Fiber dimension for 50/125 optical fiber......................................................................................... 6

3-10 Optical fiber cable............................................................................................................................ 7

3-11 Optical fiber in loose tube cable........................................................................................... 7

3-12 Optical fibers in tight buffer cable............................................................................................ 7

3-13 Fibers with offset axes....................................................................................................... 8

3-14 Off-center and elliptical cores.................................................................................................. 8

3-15 Angular misalignment of fiber axes............................................................................................... 8

3-16 Numerical aperture mismatch....................................................................................................... 8

3-17 Fiber separation loss due to spreading........................................................................................... 8

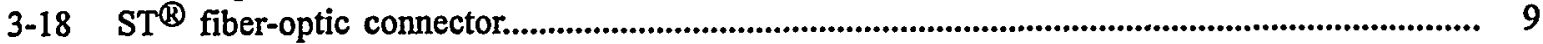

3-19 SMA fiber-optic connector.................................................................................................. 9

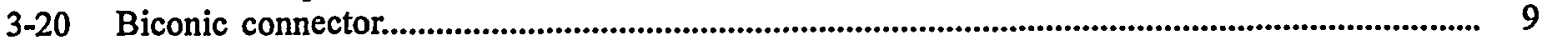

3-21 Example of clamp-type mechanical splice..................................................................................... 10

5-1 Fiber-optic transmission systems.................................................................................................. 16

5-2 CCTV alarm assessment system using coax cable................................................................... 17

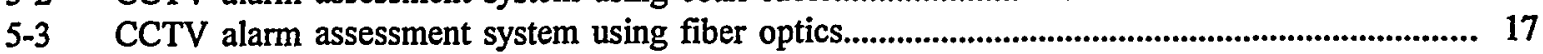

5-4 CCTV system incorporating video and control signals................................................................. 17

5-5 Wavelength division multiplexing................................................................................................ 17

5-6 Alarm communications system.................................................................................................... 18

5-7 Video/audio link using fiber optics............................................................................................. 19

5-8 Causing an alarm on an optical continuity sensor........................................................................... 19

5-9 Alarm causes for a light disturbance sensor.................................................................................. 19

5-10 Typical components of a fiber-optic light disturbance sensor........................................................... 20

5-11 Buried installation of fiber-optic intrusion detection system........................................................... 21

5-12 Treaty verification seal..................................................................................................................... 22

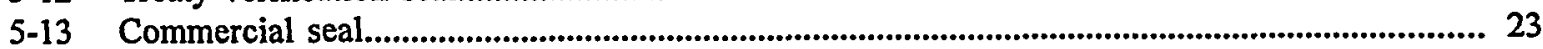

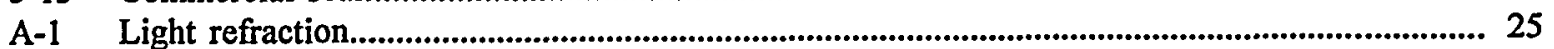

A-2 Optical fiber showing the propagation of light through the core......................................................... 25

A-3 Numerical aperture and acceptance angle.................................................................................... 26

A-4 Effects of curved fiber on light transmission............................................................................... 26

A-5 Typical fiber's attenuation as a function of wavelength...................................................................... 27

A-6 Step-index multimode fiber............................................................................................................... 27

A-7 Modal dispersion in step-index multimode fiber.................................................................................. 27

A-8 Graded-index multimode fiber...................................................................................................... 28

A-9 Correcting modal dispersion through index grading........................................................................... 28

A-10 Single-mode fiber.............................................................................................................................. 28 


\section{Acknowledgments}

The author would like to express his appreciation to the following technical experts for providing research materials and private consultation, and for reviewing the report and suggesting improvements: Bill Black, Tim Malone, Larry Miller, Dale Murray, Charles Ringler, and Bill Stewart. The author would also like to thank Priscilla Dwyer, Senior Safeguards Technical Analyst at the U.S. Nuclear Regulatory Commission, for reviewing the document and providing insightful comments about the report's intended audience. Finally, the author thanks Carol Whiddon and Colleen Lennon-Wallach of $\mathrm{BE}$, Inc., for their fine work in preparing the document for publication. 


\section{Introduction}

\subsection{Discussion of NRC Requirements}

U. S. Nuclear Regulatory Commission (NRC) regulations under part 73 "Physical Protection of Plants and Materials" of Title 10, Code of Federal Regulations, specify performance requirements for the physical protection of special nuclear materials and associated facilities. For fuel cycle facilities using or possessing a formula quantity of strategic special nuclear material, paragraph 73.45(c)(1)(iii) calls for the use of detection and surveillance subsystems and procedures to discover and assess unauthorized activities and conditions and to communicate them so that response can stop the activity or correct the conditions. For these facilities, an example reference system is outlined in section 73.46. Paragraph 73.46(e)(1) states that "[t]he licensee shall provide an intrusion alarm subsystem with the capability to detect penetration through the isolation zone and to permit response action." Paragraph 73.46(e)(8) states that "[a]ll exterior areas within the protected area shall be monitored or periodically checked to detect the presence of unauthorized persons, vehicles, materials, or unauthorized activities."
Power reactor licensees are subject to the provisions of section 73.55. Paragraph 73.55(c)(4) states that "[d]etection of penetration or attempted penetration of the protected area or the isolation zone adjacent to the protected area barrier shall assure that adequate response by the security organization can be initiated." Paragraph $73.55(\mathrm{~h})(6)$ states that "[t]o facilitate initial response to detection of penetration of the protected area and assessment of the existence of a threat, a capability of observing the isolation zones and the physical barrier at the perimeter of the protected area shall be provided, preferably by means of closed circuit television or by other suitable means which limit exposure of responding personnel to possible attack."

\subsection{Purpose of Document}

With respect to the physical protection of special nuclear material and associated facilities, applications of fiber optics include (1) communication of video, data, and voice information and (2) detection of intrusions or penetrations into controlled areas. The purpose of this report is to provide technical information useful for the development of fiber-optic communications and intrusion detection subsystems relevant to physical protection.

The discussion of equipment or systems in this document does not constitute acceptance or endorsement by the NRC. 


\section{Background}

Optical fibers are long, hair-like strands of transparent glass or plastic. Fiber optics is the class of optical technology that uses these transparent fibers to guide light from one place to another. Fiber optics has found applications in the areas of imaging, communications, and the sensing of physical parameters. Fiber-optics technology is so widely used now that the term "fiber optics" is almost as well known as "electronics."

Since the 1950s, the medical community has used optical fiber bundles, called endoscopes, to look inside the body without surgery. Endoscopes are also used to guide laser light into patients' bodies so that physicians can perform internal laser surgery.

The telecommunications industry has benefitted greatly by applying fiber optics to their communication networks. Signal speeds and traffic capacity have increased considerably, and the quality of voice signals has improved noticeably since the incorporation of optical fiber into long-distance systems. An added benefit is that fiber-optic cables are smaller and lighter than their electrical counterparts. Interference from lightning strikes and electrical grounding problems is eliminated in fiber-optic systems. Fiber optics is not limited to long-distance communication use-transmission of cable television, closed-circuit television, and computer data is currently being accomplished via fiber optics.

Finally, fiber optics has found applications in the sensing of physical properties. The transmission of light through an optical fiber is changed, sometimes minutely (but detectably), by such factors as temperature, pressure, motion, and even magnetic fields.

Several areas of fiber-optics technology are applicable to security. In particular, this report addresses those applications that use fiber optics for communications and sensing. 


\section{Fiber-Optics Technology}

Fiber optics is becoming more and more prevalent as a means of communicating information and as a means of sensing minute environmental changes (e.g., motion, vibration, and pressure). This chapter describes how optical fiber can be used to carry light signals and how it can be used to sense environmental changes. System components-cable, light sources, light detectors, connectors, and splices-are also described in this chapter.

\subsection{Theory of Operation}

The following two sections deal with the optical characteristics of fiber that (1) allow light signals to be transmitted through the fiber from a light source to a detector, and (2) permit environmental changes to be sensed.

\subsubsection{Optical Fiber Transmission Fundamentals ${ }^{1-3}$}

[Note: For a more in-depth presentation of this material, see Appendix A.]

Light is guided by an optical fiber due to its special construction. An optical fiber consists of two parts: a lightguiding core and a surrounding optical cladding (see Figure 3-1).

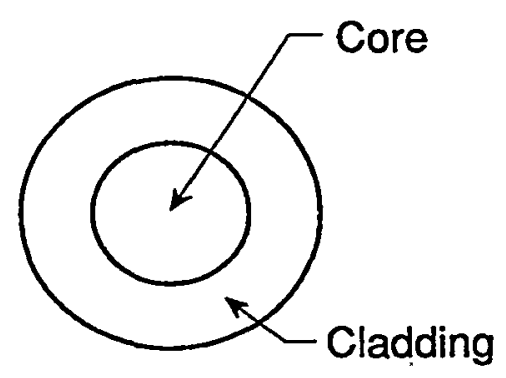

Figure 3-1. Core and cladding of optical fiber

The cladding is reflective and the core is very transparent. As light travels through the fiber, it remains in the core by bouncing off the reflective cladding. This "light pipe" confines the light and guides it along the fiber (see Figure 3-2).

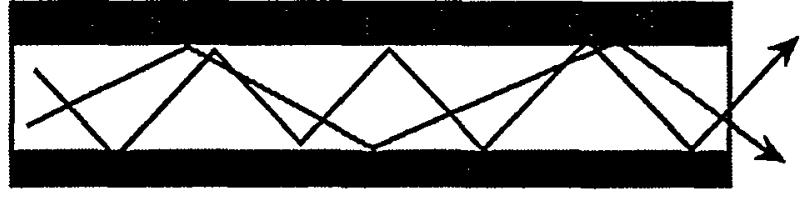

Figure 3-2. Optical fiber guides light

The fiber does not have to be straight-light will follow the fiber around a turn as long as the turn is not too tight. The allowed tightness of the turn is determined by the refractive indexes of the core and cladding. Refractive index is the measure of a material's ability to bend light.

A measure of a fiber's ability to accept light is called numerical aperture, NA. Numerical aperture is related to the broadness-of-angle over which the end of the fiber will gather light. The larger the numerical aperture, the larger the light-gathering angle.

Due to the high transparency of a glass fiber core, light can travel through the fiber several kilometers and still retain much of its brightness (optical power). To put this in perspective, compare the transparency of an optical fiber to that of a picture window found in a home. The plate glass picture window is $1 / 8$-inch thick. It could be replaced with a 3-mile thickness of optical glass fiber material and the same bright image would be seen (same transparency). Optical fiber is also made from plastic, although plastic fibers reduce optical power much more than equivalent lengths of glass fiber. Plastic fibers can be used for distances of 100 meters or less.

As light travels through an optical fiber, it loses some optical power. This reduction in power is called attenuation and is a result of impurities in the fiber and imperfections in the fiber's manufacture. Attenuation is measured in decibels (dB), which are logarithmic units commonly used to relate input power to output power. If light is reduced to $1 / 10$ of its original power while traveling from one end of the fiber to the other, the attenuation is $10 \mathrm{~dB}$. Light reduced to $1 / 100$ of its power has undergone $20 \mathrm{~dB}$ of attenuation. Similarly, if only $1 / 1000$ of the power remains, the light has been attenuated $30 \mathrm{~dB}$, and so on. A fiber's quality is often characterized in terms of its attenuation per kilometer $(\mathrm{dB} /$ $\mathrm{km})$. 
Attenuation is an important issue in the design of fiber-optic systems. In fact, all of the optical system losses should be accounted for to ensure that sufficient optical power reaches the detector. The maximum length of transmission depends on all of the system losses.

Wavelength is the color-determining property of light and plays an important part in fiber-optic systems. Most fiberoptic systems operate in the near infrared region where losses for glass fibers are at a minimum. Three operating wavelengths are commonly used: 850 nanometers $(\mathrm{nm})$, $1300 \mathrm{~nm}$, and $1550 \mathrm{~nm}$, with the longer wavelengths having the lowest loss. Plastic fiber systems operate in the visible red region at $665 \mathrm{~nm}$.

Fiber can be classified as either multimode or single-mode. From a dimensions standpoint, the core of multimode fiber is much larger than that of single-mode fiber. Multimode core diameters range from 50 to 100 microns $(0.002$ to 0.004 inches), while the core diameter of single-mode is about 8 microns ( 0.0003 inches).

As its name implies, multimode fiber allows many light modes or "rays" to be transmitted along the fiber. Originally, all multimode fiber was step-indexed, meaning that there was an abrupt change in the refractive index between core and cladding. In step-index multimode fiber, rays of light bounce off the reflective cladding at different angles (see Figure 3-3).

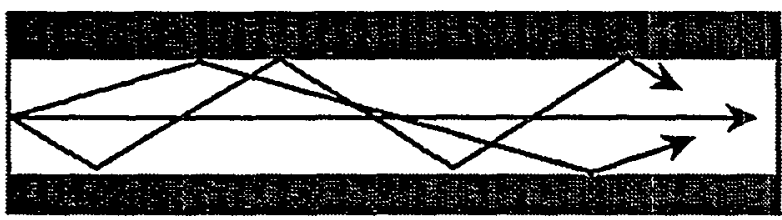

Figure 3-3. Step-index multimode fiber

From the book Understanding Telecommunications and Lightwave Systems: An Entry-Level Guide, by John G. Nellist, O1992. Published by IEEE Press. Used by permission of publisher.

Due to the reflection angle differences, some rays travel longer distances than others, and they arrive at the far end of the fiber later than those rays taking shorter paths. Light signals (which are made up of all of these rays) tend to get "smeared" in time as they travel down the fiber. This smearing is called modal distortion, and it reduces the usable length of step-index multimode fiber, although the actual usable length also depends on how fast the signal is changing (i.e., the signal's bandwidth). Index grading (gradual changing of the refractive index from core to cladding) is an improvement that attempts to equalize the times at which the light rays arrive at the far end, resulting in less modal distortion. This improved multimode fiber is called graded index. See Figure 3-4.

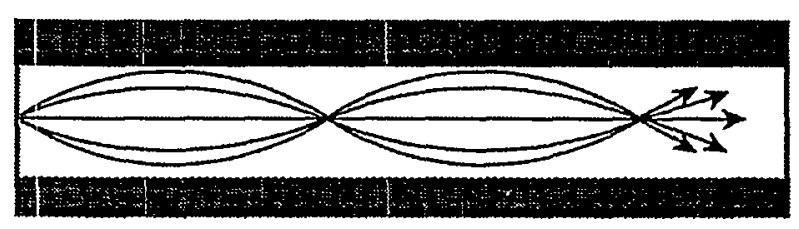

Figure 3-4. Graded-index multimode fiber

From the book Understanding Telecommunications and Lightwave Systems: An Entry-Level Guide, by John G. Nellist, O1992. Published by IEEE Press. Used by permission of publisher.

Over moderate distances (a few kilometers) and at moderate signal rates, graded-index multimode fiber performs satisfactorily. But over long distances some modal distortion still occurs.

The only way to completely eliminate modal distortion is to use single-mode fiber. In single-mode fiber, only a single ray is allowed to pass through the core (see Figure 3-5).

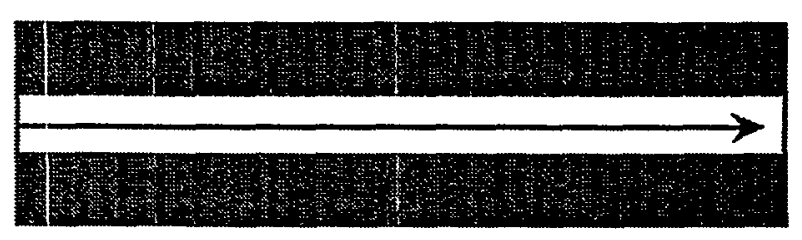

Figure 3-5. Single-mode fiber

From the book Understanding Telecommunications and Lightwave Systems: An Entry-Level Guide, by John G. Nellist, (01992. Published by IEEE Press. Used by permission of publisher.

In single-mode fiber, no smearing occurs and modal distortion is eliminated. Single-mode fiber allows signals to be sent over very long distances (tens of kilometers) and at very high signal rates.

\subsubsection{Sensing Phenomena ${ }^{4,5}$}

Fiber-optic systems can be used to sense many physical parameters. Light intensity, displacement, position, temperature, pressure, sound, and vibration are some of the physical phenomena that fiber optics can be used to sense. In intrusion detection systems, the primary sensing phenomena used are displacement, pressure, and vibration, which are all closely related. Actual fiber breakage is also used as a detection event. 
Fiber-optic sensors can be grouped into two broad categories: (1) phase-modulated sensors, and (2) intensitymodulated sensors. Intensity-modulated sensors detect variations in light intensity caused by the physical environment. Phase-modulated sensors use light beam-splitting and comparison methods to detect phase changes in the light induced by the physical environment.

Intensity-modulated sensing can be as simple as detecting a break in the fiber. This is the light/no-light condition that is used in security systems that detect the cutting of a webbing that contains fiber. Another intensity-modulated sensing mechanism important to fiber systems involves microbending. Special plates impose microbends in the fiber when pressure is applied. As a fiber is bent, some light is lost (see Figure 3-6). A significant change in light intensity at the detector indicates an alarm.

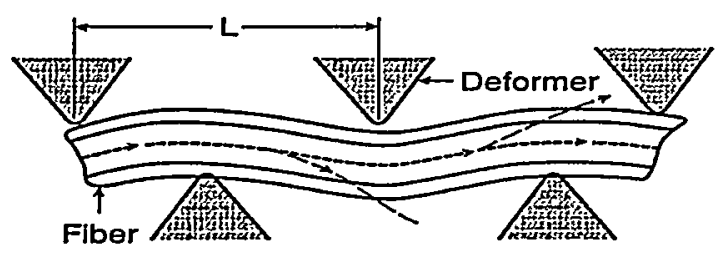

The deformation and resulting coupling of light from core to cladding that occurs in a microbend intensity-type fiber-optic sensor.

\section{Figure 3-6. Effects of microbending on fiber}

From Fiberoptic Sensor Technology Handbook by Charles $M$. Davis, et al., O1986. Published by Optical Technologies, Inc. Used by permission of publisher.

Sensors mounted on fences or buried in the ground can make use of this concept. Sensing changes in the light intensity due to microbends induced by displacement, pressure, or vibration provides a way to detect walking intruders or intruders climbing a fence.

Phase modulation uses interferometry to detect changes in the phase of the light and requires a laser source. Interferometry involves splitting the light into two paths: a reference path and a sensing path. At the detector, light from the two paths is recombined and forms an interference pattern. Moving or flexing the fiber-optic cable causes changes in the light path length, which disturbs the light. This disturbance is detectable as an alarm.

Some sensors use changes in speckle patterns to detect pressure/displacement. As light travels along the fiber, the various modes interfere with each other and form a speckle pattern at the end of a fiber (Figure 3-7).

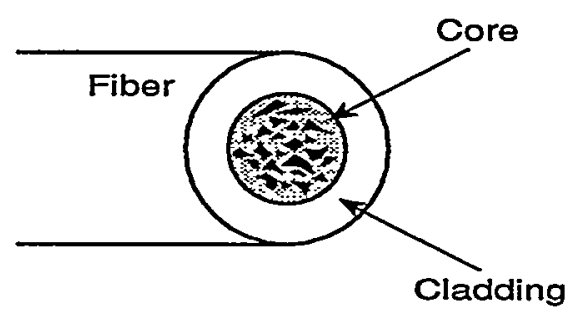

Figure 3-7. Speckle pattern

From Fiber Optics Handbook, 2nd ed., by Christian Hentschel, O1988. Published by Hewlett-Packard Company. Used by permission of publisher.

Of course, multimode fiber must be used to obtain modal interference. Graded-index fibers are preferable for this kind of detection system, since they show more pronounced speckle patterns than step-index fibers. ${ }^{6}$ This kind of system requires a laser source.

\subsection{System Components ${ }^{2,3}$}

A fiber-optic system-whether it is used for communication or sensing - consists of three basic elements: the emitter (light source), the optical fiber (and its associated cable), and the detector (light receiver). See Figure 3-8.

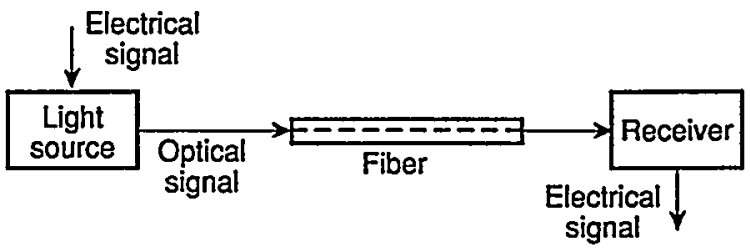

Figure 3-8. A basic fiber-optic system

From the book Understanding Fiber Optics, by Jeff Hecht, (01987. Published by SAMS Publishing, a division of Prentice Hall Computer Publishing. Used by permission of publisher.

The emitter, fiber, and detector are joined by special fiberoptic connectors. Pieces of fiber can be joined by connectors or more permanent splices. The following four sections describe each of these components in more detail.

\subsubsection{Optical Fiber and Cable}

A variety of optical fibers exist-from the single-mode glass (silica) fibers used in high-speed long-distance communications to the large-diameter plastic fibers used for short-distance lighting and imaging applications. Fiber types may be categorized by: 
(1) composition (e.g., silica or plastic)

(2) modal characteristics (multimode or single-mode)

(3) index characteristics (step-index or graded-index)

The dimension of most fiber (core and cladding) is on the order of that for human hair-about 0.004 inches $(=100$ $\mu \mathrm{m})$. Optical fiber diameters are usually stated in microns $(\mu \mathrm{m})$, as a ratio of core diameter to cladding diameter. For example, 50/125 means a 50- $\mu \mathrm{m}$ diameter core and a 125$\mu \mathrm{m}$ diameter cladding (Figure 3-9).

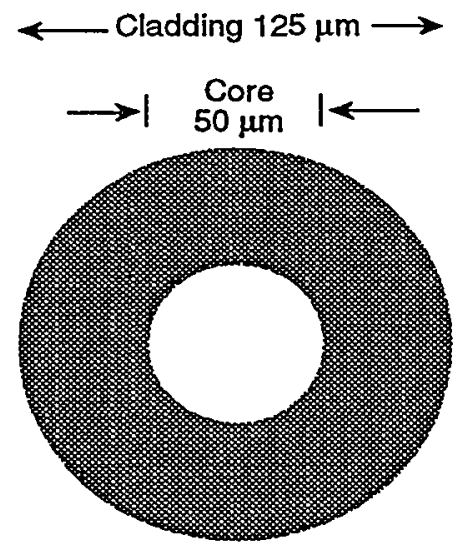

Figure 3-9. Fiber dimension for 50/125 optical fiber

Common multimode glass fibers are $50 / 125,62.5 / 125$, and $100 / 140$. Single-mode glass fibers have much smaller cores-8.3/125, for example. A plastic coating, called the buffer, is applied to the cladding to protect the fiber from scratches and to give added strength. Buffer diameters are $250 \mu \mathrm{m}$ or $900 \mu \mathrm{m}$, depending on the cabling method and whether the fiber is to be used in outdoor or indoor applications. Table 3-1 shows characteristics of several glass-core/ glass-cladding (i.e., "all" glass) fiber types.
The three varieties of multimode fiber in the table are typically used at the $850-\mathrm{nm}$ and $1300-\mathrm{nm}$ wavelengths and are usable up to several kilometers. The 8.3/125 singlemode fiber is designed for $1310-\mathrm{nm}$ and $1550-\mathrm{nm}$ use and serves exceptionally well in long-distance communication links up to $50 \mathrm{~km}$ without the requirement for signal regeneration.

In addition to all-glass fibers, plastic-clad glass and allplastic fibers are also used. The core dimension of plasticclad glass fiber ranges from $200 \mu \mathrm{m}$ to $1000 \mu \mathrm{m}$.

Attenuation for plastic-clad is about double that of all-glass fiber-about $10 \mathrm{~dB} / \mathrm{km}$, which limits its use to lengths less than $2 \mathrm{~km}$. One advantage of plastic cladding is that the required refractive index difference between the core and the cladding is easy to attain in manufacture-plastic inherently has a lower refractive index than glass. On the contrary, all-glass fiber uses a complex, multi-step doping process to sufficiently lower the refractive index of the cladding. Plastic-clad glass fiber is operated in the 820-to850-nm range.

All-plastic fiber typically has a diameter of $1000 \mu \mathrm{m}$ or larger. Attenuation of several hundred decibels per kilometer is common, limiting its usefulness to less than 100 meters. All-plastic fiber performs optimally at a wavelength of about $665 \mathrm{~nm}$.

The larger core plastic-clad and all-plastic fibers do have the ability to gather light more easily than the small-core allglass fibers, which simplifies the task of coupling light sources to the fiber.

For practical use, optical fiber is placed in protective cables. Many possible combinations of components-strengthening members (e.g., steel, kevlar), jacketing materials, numbers

Table 3-1. Common fiber characteristics

\begin{tabular}{lcccccccc}
\hline $\begin{array}{c}\text { Fiber } \\
\text { type }\end{array}$ & Mode & $\begin{array}{c}\text { Core } \\
\text { diameter } \\
(\mu \mathrm{m})\end{array}$ & $\begin{array}{c}\text { Cladding } \\
(\mu \mathrm{m})\end{array}$ & $\begin{array}{c}\text { Coating } \\
\text { diameter } \\
(\mu \mathrm{m})\end{array}$ & $\begin{array}{c}\text { Refractive } \\
\text { index delta }\end{array}$ & $\begin{array}{c}\text { Numerical } \\
\text { aperture }\end{array}$ & $\begin{array}{c}\text { Max. } \\
(\mathrm{dB} / \mathrm{km})\end{array}$ & $\begin{array}{c}\text { BW-length } \\
\text { product } \\
(\mathrm{MHz}-\mathrm{km})\end{array}$ \\
\hline $50 / 125$ & multimode & 50 & 125 & 250 & $1.0 \%$ & 0.20 & $4.00^{1}$ & 500 \\
$62.5 / 125$ & multimode & 62.5 & 125 & 250 & $2.0 \%$ & 0.275 & $3.75^{1}$ & 160 \\
$100 / 140$ & multimode & 100 & 140 & 250 & $2.2 \%$ & 0.29 & $5.00^{1}$ & 100 \\
$8.3 / 125$ & single-mode & 8.3 & 125 & 250 & $0.37 \%$ & 0.13 & $0.40^{2}$ & NA $^{3}$ \\
\hline
\end{tabular}

At $850 \mathrm{~nm}$

${ }^{2}$ At $1310 \mathrm{~nm}$

${ }^{3}$ Source dependent. Chromatic dispersion is the bandwidth limiting factor in single-mode fiber. 
of fibers-exist to address the diverse applications of optical fiber. Cables may have from 1 to over 100 fibers. Figure 3-10 shows an example of a fiber-optic cable.

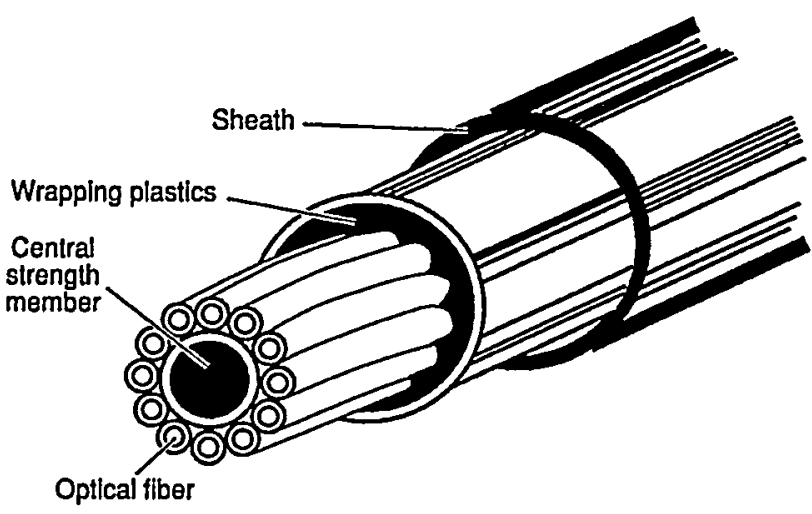

Figure 3-10. Optical fiber cable

From the book Understanding Telecommunications and Lightwave Systems: An Entrv-Level Guide, by John G. Nellist, O1992. Published by IEEE Press. Used by permission of publisher.

There are two basic types of fiber-optic cable: loose tube and tight buffer. In loose tube cable, the silica fiber is coated (buffered) with plastic to a diameter of $250 \mu \mathrm{m}$ and lies loosely in a tube. The tube has a much larger diameter than the buffered fiber to allow the fiber to be installed in a loose spiral. Thus, the fiber can move freely with respect to the tube. This construction technique isolates the fiber from external stresses applied to the cable during installation. It also relieves stresses on the fiber imposed by temperature expansion/contraction. See Figure 3-11.

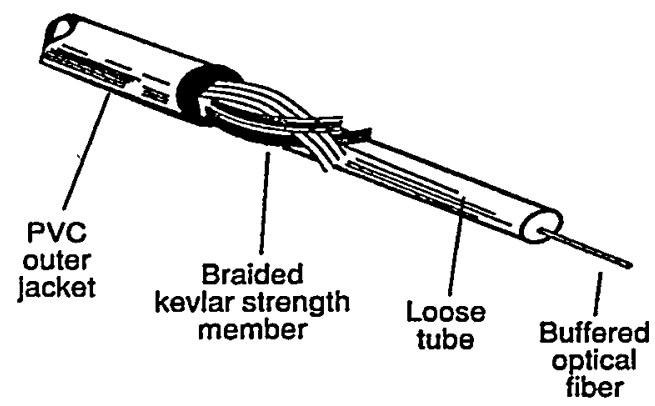

Figure 3-11. Optical fiber in loose tube cable

From Math Associates, Inc., fiber optics product catalog, OSeptember 1990. Used by permission of Maih Associates, Inc.

The tube may be filled with gel to keep moisture out, to lubricate the fibers so that they can move more easily, and to improve crush resistance. Tubes may contain up to 12 fibers. Several tubes are usually contained in one cable.

In tight buffer cable, the silica fiber is buffered with one or more plastic layers, usually to a diameter of $900 \mu \mathrm{m}$ (Figure 3-12).

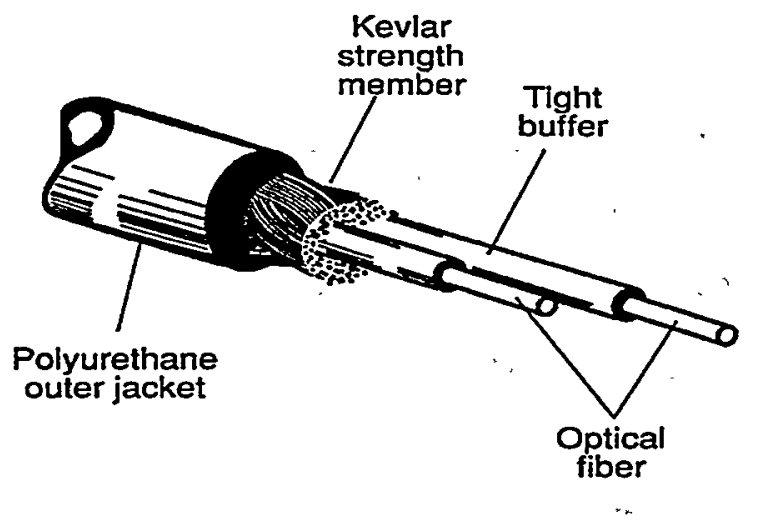

Figure 3-12. Optical fibers in tight buffer cable

From Math Associates, Inc., fiber optics product catalog, OSeptember 1990. Used by permission of Math Associates, Inc.

Inner buffering layers are often soft so that they will deform with external pressure. This alleviates stress on the fiber. The outer buffering is a hard plastic layer. Several buffered fibers may be contained in this kind of cable.

\subsubsection{Connectors and Splices}

Connectors and splices are the means by which the various components of a fiber-optic system can be linked together. Connectors are devices that allow the easy connection and disconnection of fiber, transmitters, and detectors. Splices are permanent connections that join two fibers.

Before describing the various kinds of connectors and splices, we will discuss attenuation at these junctures and the various causes of it. Of course, the desire is to keep attenuation at a minimum in all light transmission elements. Attenuation in fiber junctures stems from fiber axis offset, off-center and elliptical cores, axis angular misalignment, numerical aperture mismatches, fiber spacing, and reflection at the fiber ends.

It is easy to see that if the two fibers' cores are not aligned and matched, some of the light will pass from the transmitting fiber's core to the receiving fiber's cladding. For example, consider Figure 3-13 where the two fiber axes are offset. 


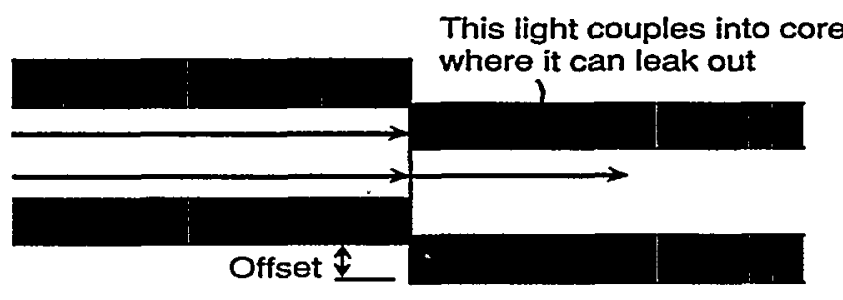

Figure 3-13. Fibers with offset axes

From the book Understanding Fiber Optics, by Jeff Hecht, (01987. Published by SAMS Publishing, a division of Prentice Hall Computer Publishing. Used by permission of publisher.

This offset-axes situation represents a loss of light from transmitting fiber to receiving fiber. Off-center and elliptical cores also result in light lost in the cladding (Figure 3-14). a. Elliptical cores

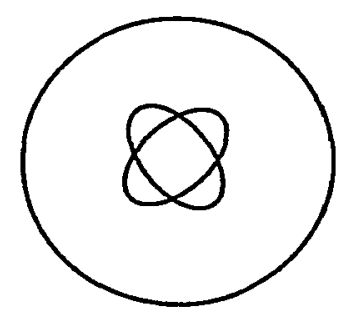

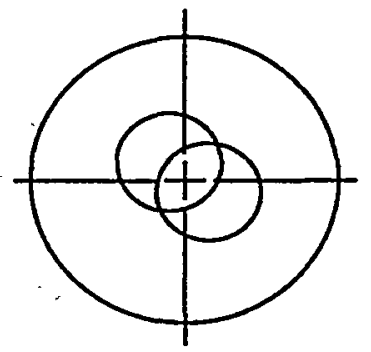

b. Off-center cores
Figure 3-14. Off-center and elliptical cores

From the book Understanding Fiber Optics, by Jeff Hecht, (01987. Published by SAMS Publishing, a division of Prentice Hall Computer Publishing. Used by permission of publisher.

A related light-loss problem occurs if there is an angle between the axes of the two fibers. This is similar to the losses experienced in fiber that is bent in too small a radius. See Figure 3-15.

If the receiving fiber has a smaller NA than the transmitting fiber, some of the light traveling out of the transmitting fiber will not be supported in the receiving fiber because its critical angle is greater. Light is again lost in the cladding. This is called numerical aperture mismatch and is illustrated in Figure 3-16.

Light spreads out as it leaves a fiber. If space exists between the two fibers, the spreading portion of the light is lost outside the core. This light-spreading loss depends on the spacing between the fibers and on the NA of the fibers (Figure 3-17).

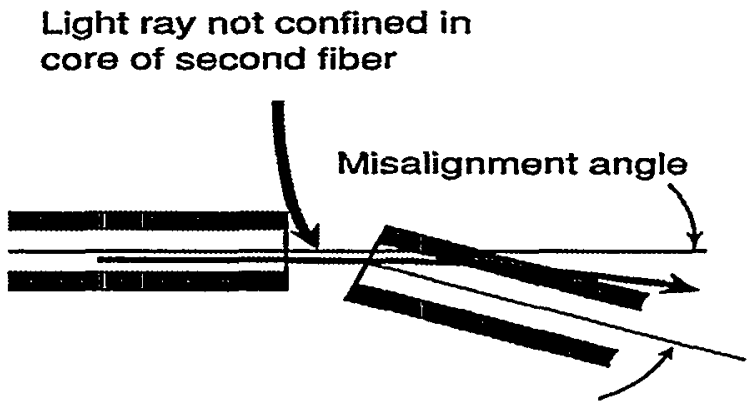

Figure 3-15. Angular misalignment of fiber axes

From the book Understanding Fiber Optics, by Jeff Hecht, (01987. Published by SAMS Publishing, a division of Prentice Hall Computer Publishing. Used by permissioin of publisher.

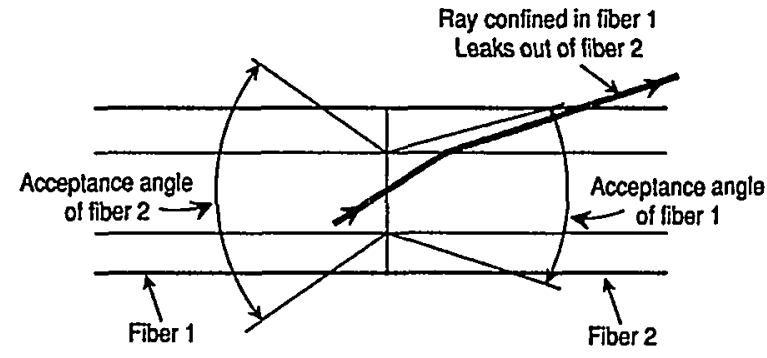

Figure 3-16. Numerical aperture mismatch

From the book Understanding Fiber Optics, by Jeff Hecht, (01987. Published by SAMS Publishing, a division of Prentice Hall Computer Publishing. Used by permission of publisher.

Light here does not enter core of receiving fiber

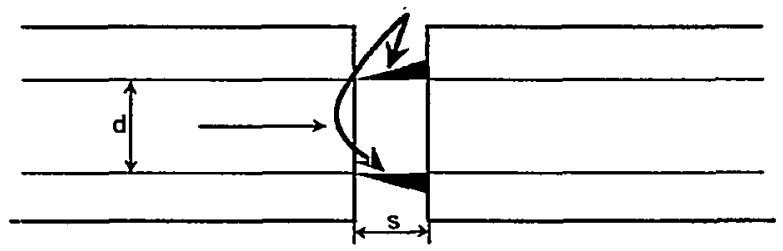

Figure 3-17. Fiber separation loss due to spreading

From the book Understanding Fiber Optics, by Jeff Hecht, (01987. Published by SAMS Publishing, a division of Prentice Hall Computer Publishing. Used by permission of publisher.

Spaced fibers also suffer from end-reflection loss, which occurs for all optically transparent materials. This reflection loss depends on the refractive indexes of the fiber and the gap. Light experiences a $3.6 \%$ reflection loss leaving the transmitting fiber and another $3.6 \%$ loss entering the 
receiving fiber (total loss of $7.2 \%$ ). This translates to a 0.32 $\mathrm{dB}$ total reflection loss due to the spacing. This is in addition to the light-spreading loss described in the previous paragraph. Reflection losses can be reduced by using antireflective coatings on the fiber ends or by filling the gap with index-matching gel.

Other losses occur (1) if the fiber ends are not cut perpendicular to the fiber axis, (2) if the end surfaces are not smooth, or (3) if dirt is present on the end of the fibers. Properly installed connectors typically have less than 0.5 dB losses.

Commonly used fiber-optic connectors are the ST, SMA, and biconic connectors. The ST ${ }^{\circledR}$ (registered trademark of AT\&T) connector is a bayonet type that utilizes a "twistlock" installation action similar to the well-known BNC electrical coaxial connector. Circular orientation of the fiber is maintained each time a connection is made due to the keying, which ensures repeatability. Figure 3-18 shows the ST connector.

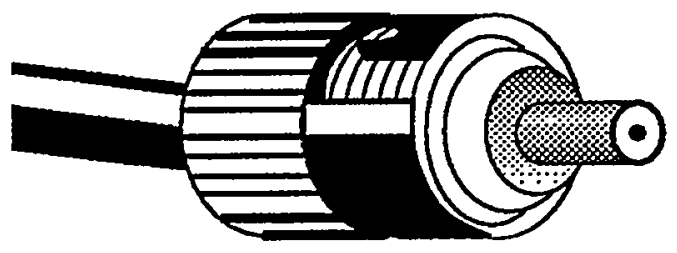

Figure 3-18. ST ${ }^{\circledR}$ fiber-optic connector

From the book Understanding Telecommunications and Lightwave Sustem.s: An Enirv-Level Guide, by John G. Nellist, O1992. Published by IEEE Press. Used by permission of publisher.

The SMA connector has similar dimensions to its SMA electrical coaxial counterpart. It utilizes a coupling nut to secure the fiber connection (similar to the familiar $F$ connector found in cable television). Circular orientation may change each time a connection is made. Because of the random orientation, and because of connector and installation imperfections, inconsistencies in the measured loss of the connector can occur from one mating to the next. Another concern is that the coupling nut can vibrate loose. Figure 3-19 shows the SMA connector.

The biconic connector is a high-performance connector used in high-speed telecommunications networks. It is designed to bring two fibers into physical contact with each other, minimizing the losses that accompany gaps between the fibers. Figure 3-20 shows the biconic connector.

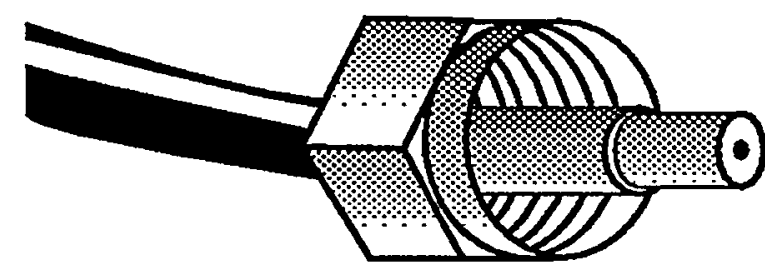

Figure 3-19. SMA fiber-optic connector

From the book Understanding Telecommunications and Lightwave Systems: An Entrv-Level Guide, by John G. Nellist, O1992. Published by IEEE Press. Used by permission of publisher.

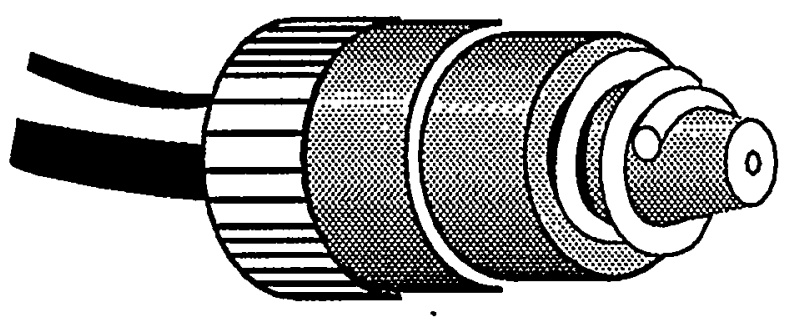

Figure 3-20. Biconic connector

From the book Understanding Telecommunications and Lightwave Systems: An Entry-Level Guide, by John G. Nellist, (O1992. Published by. IEEE Press. Used by permission of publisher.

Splices are intended to permanently join two fibers. Splicing is required when two or more lengths are to be joined to make one continuous fiber (e.g., for long-distance applications) or if a repair is to be made to a broken fiber. Splices should be low-loss and field-installable. Field installation requires that alignments are easily performed, that specialized tools be kept to a minimum, and that the splice can be completed rapidly.

There are two basic types of splices: fusion and mechanical. Fusion splicing, which requires more expensive equipment, is the welding together of two fibers. For silica fibers, this is accomplished by heating the fibers to their melting point (about $2000^{\circ} \mathrm{C}$ ) with an electric arc. Prior to actually fusing the fibers, the cables and fibers are prepared: the cable jacketing, kevlar, and buffering materials are removed; the fibers are scribed and broken to produce smooth, perpendicular fiber end surfaces; and the fibers are aligned. Alignment is checked visually with a microscope or by monitoring light transmission through the aligned fibers. After the fibers are aligned, the fibers are fused. The attenuation of a fusion splice is typically less than $0.1 \mathrm{~dB}$. The connection is then placed in a closure (essentially a small box), which provides environmental protection and 
mechanical support for the splice and a convenient place to neatly store excess fiber. Since these operations are usually done in a splicing van, the resulting cable slack and splice closures are stored in a cable enclosure for protection.

Two kinds of mechanical splices are used: the epoxy type and the clamp type. Attenuation of mechanical splices is somewhat more than the fusion type (about $0.25 \mathrm{~dB}$ ), but less equipment is required.

One example of a clamp splice consists of (1) a clear plastic housing with a lens molded into its underside to enhance viewing for a more precise splicing, (2) a glass capillary, prefilled with an index-matching material, and (3) a metal spring, which has a compliant member to accommodate size differences in the fiber and to protect the fibers from the spring. The stripped and cleaved fibers are inserted into the housing and guided into the capillary where they butt in the center. The spring is engaged and latched into a detent position to align and hold the fibers in place. See Figure 3-21 for an example of this kind of splice.

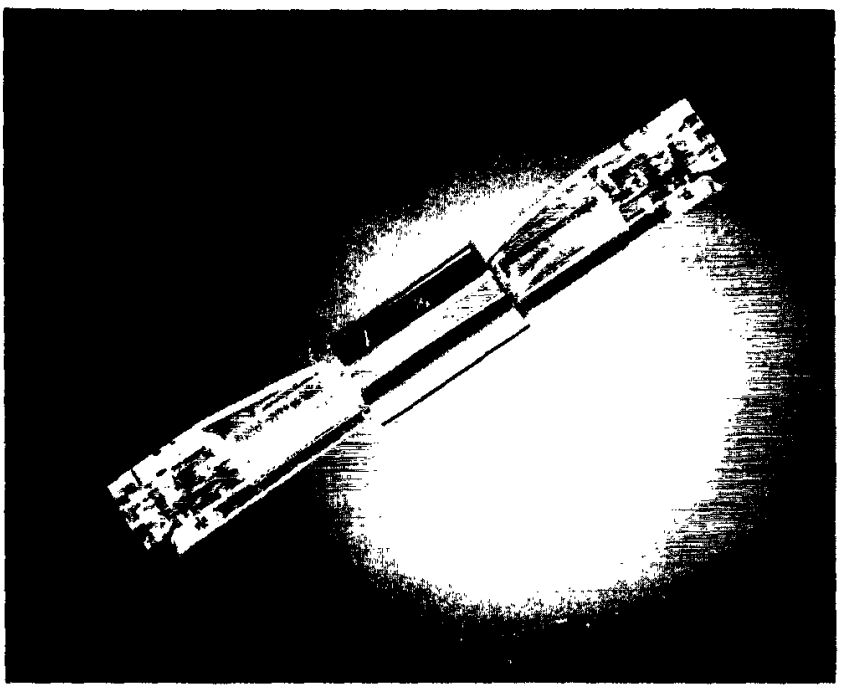

Figure 3-21. Example of clamp-type mechanical splice

From AT\&T Network Cabling Systems CSL LightSplice ${ }^{\text {TM System }}$ Data Sheet, 01990. Used by permission of AT\&T Network Cabling Systems.

One epoxy-type splice is the elastomeric splice. Epoxy is inserted into the splice housing. Then the fiber ends are guided into tapered holes in the splice housing and into a small triangular-shaped groove until they meet at the center. Finally, the epoxy is cured with ultraviolet light. The use of ultraviolet-curable epoxy lessens the splicing time as compared to normal epoxy.

\subsubsection{Emitters (Light Sources)}

Emitters generate the light beams in a fiber-optic system. They convert electrical information-carrying signals to optical signals. Two kinds of emitters are used in fiber-optic systems: the light-emitting diode ( $L E D)$ and the brighter laser diode. The decision of whether to use an LED or a laser diode in a fiber-optic system depends on output power (brightness), operating wavelength, spectral width, modulation bandwidth, component lifetime, and cost.

LEDs are often selected as fiber-optic light sources for three reasons. First, they are less costly than laser diodes. Second, their optical output is linear with respect to input current, which makes them usable for both analog and digital transmission. Third, their lifetime is much longer than laser diodes. The optical output of an LED is typically $-15 \mathrm{dBm}$ $(30 \mu \mathrm{W})$. LEDs are used at $665 \mathrm{~nm}$ (plastic fiber use), $850 \mathrm{~nm}$, and $1300 \mathrm{~nm}$, but rarely at $1550 \mathrm{~nm}$, due to the more complex manufacturing process required to produce LEDs that operate at $1550 \mathrm{~nm}$.

Some applications require the use of laser diodes. To transmit over long distances, the greater output power (brightness) of a laser diode is needed. Outputs of +10 $\mathrm{dBm}(10 \mathrm{~mW})$ are common for laser diodes. Laser diodes also have narrower spectral widths than LEDs, which means less chromatic dispersion and hence permits longer transmission distances. The nonlinear input/output characteristic of laser diodes makes them less suitable for analog transmission applications. Laser diode lifetimes are shorter than those of LEDs, since they are operated at higher currents to obtain the higher output power. This causes thermal fatigue and degradation of performance, eventually causing laser diodes to fail. Laser diodes are typically used at $1300 \mathrm{~nm}$ and $1550 \mathrm{~nm}$.

\subsubsection{Detectors (Light Receivers)}

Detectors are the light-beam receivers in a fiber-optic system. They convert optical information-carrying signals to electrical signals. Two types of detectors typically used in fiber-optic systems are the PIN diode and the avalanche photodiode (APD). A detector is selected based on performance (e.g., sensitivity, detection wavelengths, dark current limitations) and cost. 
PIN diodes used in fiber-optic systems have a window to allow light to enter. The light causes an electrical current to flow through the diode. The efficiency of converting light to electrical current is not high for a PIN diode, so the electrical signal is amplified.

APDs have higher light-to-electrical efficiency (sensitivity) than PIN diodes due to a photomultiplication effect that occurs during their operation. Thus, an amplifier is not required. Two kinds of APDs are common. Germanium (Ge) APDs suffer from excessive thermally induced dark current (electrical current when no light is present). Thus, they require cooling to about $20^{\circ} \mathrm{C}$ or $25^{\circ} \mathrm{C}$ for acceptable performance. A newer indium gallium arsenide (InGaAs) APD has lower dark current, so cooling is not required. The InGaAs device also performs better at $1550 \mathrm{~nm}$ than other detectors.

\subsection{References}

1. Coden, Michael H., "Everything You Ever Wanted to Know About Fiber Optics and LANs, But Were Afraid to Ask!" The Fiber Optic LAN Handbook, 4th ed., Codenoll Technology Corporation, Yonkers, New York, October 1991, pp. 38-43.

2. Hecht, Jeff, Understanding Fiber Optics, Howard W. Sams and Company, Indianapolis, Indiana, 1987.

3. Nellist, John G., Understanding Telecommunications and Lightwave Systems: An Entry-Level Guide, IEEE Press, New York, 1992, pp. 6, 106-173.

4. Krohn, D. A., Fiber Optic Sensors: Fundamentals and Applications, 2nd ed., Instrument Society of America, Research Triangle Park, North Carolina, 1992.

5. Davis, Charles M., et al., Fiberoptic Sensor Technology Handbook, Optical Technologies, Inc., Herndon, Virginia, 1986.

6. Hentschel, Christian, Fiber Optics Handbook, 2nd ed., Hewlett-Packard, January 1988, pp. 117-118. 


\section{Systems Engineering}

\subsection{Specification}

To specify an appropriate fiber-optic intrusion detection system, the designer should consider how it fits into the overall physical protection system. The following items influence the effectiveness of any intrusion detection system and so should be considered when specifying a fiber-optic intrusion detection system:

(1) Facility threats and targets

(2) Peculiarities of the facility

(3) Other detection and assessment elements

(4) Delay and response mechanisms

In addition, issues that are more directly related to the intrusion detection system should be understood. These include the system's probability of detection, invalid alarm rate, covertness, susceptibility to vandalism, reliability, ease of maintenance, and cost (both of installation and of operation). Manufacturers can provide information that addresses these issues. The degree to which the system protects itself from a malevolent insider should also be considered. Deciding whether to install a fence-mounted or a stand-alone system, a buried or a submergible system, a wall-mounted or a wall-encased system depends on the user's requirements and the peculiarities of the specific facility. Analyses and decisions should be made on a facility-by-facility basis.

Specifying a fiber-optic communication system includes selecting appropriate (1) operating wavelength, (2) transmitters and receivers, (3) fiber and cable type, (4) splices and connectors, and (5) closures and cross-connection boxes to meet the requirements of the user and to allow for expansion and upgrading.

The choice of which operating wavelength to use is influenced by the data-speed, distance, and cost requirements. Shorter wavelength systems $(850 \mathrm{~nm})$ are less expensive but can not transmit data as far or as fast as the 1300- and 1550-nm systems; 850-nm and 1300-nm systems are expected to meet the requirements of most safeguards applications. Plastic fiber systems $(665 \mathrm{~nm})$ are also available, but their usefulness is usually limited to less than 100 meters.

Selection of the wavelength also influences the choice of the transmitter. Transmitters operating at 850 and $1300 \mathrm{~nm}$ use LEDs or laser diodes, whereas $1550-\mathrm{nm}$ transmitters require a laser diode. Laser diodes provide more power, but have shorter lifetimes and are more expensive than LEDs.

Receivers use either a PIN diode or an avalanche photodiode (APD). PIN diode receivers are less sensitive, but are adequate for most anticipated safeguards applications (i.e., short-to-moderate distance applications). PIN diode receivers are also less expensive. For applications requiring increased receiver sensitivity, an APD receiver may be used.

Specifying the type of fiber to be used requires selection of (1) the fiber material (plastic or glass), (2) the fiber size, and (3) multimode or single-mode. Appropriate choices depend on data-speed and distance requirements. It is not likely that single-mode fiber would be required for safeguards applications.

Cable selection should consider (1) fiber type and size, (2) fiber count, (3) loose tube or tight buffering, (4) strength member material, (5) armoring material, and (6) jacket type to suit the intended environment (indoor or outdoor), flame retardancy, etc. In addition, the cable should be suited to its placement method (overhead, direct burial, conduit, duct, tray, riser, plenum, or wall). Consideration should be given to pulling strength (important during installation), minimum bend radius, weight and size, crush strength, and color coding of fibers. ${ }^{1}$

Splices and connectors should be selected based on their ease of installation, amount of loss, loss stability over temperature, and durability. In addition, connectors should be small and should have good loss repeatability over many hundreds of mating cycles. Connectors with a bayonet latch are desirable for easy on-off and for their secure connections. 1

Closures (protective housings for splices) should be small, but should allow for system growth. Closures should be dustproof and, if used outdoors, waterproof. Crossconnection boxes - which are used to mount connectors and allow flexible and easy rearrangement of optical circuitsshould also be small, allow for growth, and ensure easy access to the patch panel. ${ }^{1}$

\subsection{Design}

Design of fiber-optic intrusion detection sensors is primarily the responsibility of the manufacturer. The manufacturer makes the selection of the sensing 
technique, cable and fiber types, light source (whether LED or laser diode), light detector, and processing equipment. The user is responsible for designing the physical layout of the sensing cable (burial, fence-mounting, etc.), the placement of control and processing equipment, and for ensuring the sensor's proper integration with other security system elements. In essence, the user buys a complete intrusion detection sensor from the manufacturer and adapts it to his particular site and requirements, receiving guidance from and working closely with the manufacturer.

Of course, turnkey fiber-optic communications systems are also available and can be purchased from systems design firms. On the other hand, fiber-optic communication systems are often designed (at the subsystem level) by the user. From his requirements, the user makes decisions about which subsystem components to use: light source, detector, fiber, cable, etc. This section will cover several basic design concepts needed to properly select subsystem components of a fiber-optic communications system.

An important concept to understand in designing a fiberoptic communications system is power budgeting. ${ }^{1-3}$ The fiber-optic system should be designed to ensure that enough optical power reaches the light receiver to be properly detected. A certain amount of power is transmitted into the fiber by the source (transmitter). Losses in the fiber, connectors, and splices reduce the power that arrives at the detector. If the power arriving at the detector is not enough to be properly detected, the power budget has been exceeded. The design should include extra margin so that the delivered power is not at the detector's minimum operating level. This loss margin allows for component variations, age degradation of components, and system repairs and additions.

Power budget is stated in terms of $\mathrm{dB}$ (decibels). It is the difference between the power transmitted into the fiber and the receiver's sensitivity (required receiver power). The sum of all the system losses and the loss margin should not exceed the power budget:

\section{Power budget $(\mathrm{dB})=$ Transmitter power - Receiver sens. $\geq$ Losses + Loss margin}

In the above equation, transmitter power and receiver sensitivity are expressed in $\mathrm{dBm}, \mathrm{a} \mathrm{dB}$ unit that references power to 1 milliwatt.

A quick power budget example: Suppose the transmitter power is $-13 \mathrm{dBm}$ and the receiver sensitivity is $-30 \mathrm{dBm}$. Then the sum of the losses (fiber, connectors, splices) and the loss margin should be less than or equal to $17 \mathrm{~dB}(-13$ $\mathrm{dBm}-(-30 \mathrm{dBm}))$.
Let's take a more detailed look at the system losses and loss margin, and then consider another example.

There are significant losses associated with coupling light from the transmitter into the fiber. This is especially true for LED transmitters, due to their large emitting surface, their wide emission angle, and the relatively small diameter of the fiber's core. Much of the light is lost in the cladding of the fiber. Laser diodes couple more light into fibers-they have a smaller emitting area, a smaller beam spread, and higher output power.

From the user's standpoint, knowing how much power is coupled into the fiber is more useful than knowing the transmitter's total output power. That is why manufacturers sometimes state how much power is actually coupled into the fiber for a given fiber size. For example, the manufacturer may specify that a particular transmitter will couple $12.5 \mathrm{dBm}$ of power into a $100 / 140$ fiber, or $-18.5 \mathrm{dBm}$ into $50 / 125$ fiber.

Fiber loss is easily calculated from the attenuation per kilometer $(\mathrm{dB} / \mathrm{km})$ specified by the manufacturer. Simply multiply the fiber's $\mathrm{dB} / \mathrm{km}$ by the length to be used to obtain the fiber loss. For example, suppose the fiber's attenuation per kilometer is $5 \mathrm{~dB} / \mathrm{km}$ and the length to be used is 400 meters $(0.4 \mathrm{~km})$. Then the loss due to the fiber is $2 \mathrm{~dB}(5 \mathrm{~dB} / \mathrm{km} \times 0.4 \mathrm{~km})$.

Losses associated with coupling light from the fiber to the receiver are generally small and can be ignored. However, receiver sensitivity (the minimum power that a receiver can detect) is important in figuring the power budget and is stated in $\mathrm{dBm}$ (e.g., $-30 \mathrm{dBm})$.

Connector and splice losses should be accounted for in power budgeting. Properly installed connectors have losses less than $0.5 \mathrm{~dB}$. Fusion splices have typically about $0.1 \mathrm{~dB}$ of loss, mechanical splices about $0.25 \mathrm{~dB}$. Manufacturers specify typical and maximum losses for connectors and splices - there is some uncertainty in the actual loss. This uncertainty depends on the quality of manufacture and the quality of installation.

Finally, loss margin should be included in the design. This safety factor allows for uncertainties in counting the losses. Loss margin can range from 3 to $10 \mathrm{~dB}$, depending on performance requirements, future additions to the systems, ease of repair, and cost.

Suppose that a $10 \mathrm{Mbit} / \mathrm{sec}, 850$-nm optical data link is to be established between two locations 1500 meters apart, using the following components and design criteria. Is the power budget sufficient? 
Transmitter power to $50 / 125$ fiber: $-18.5 \mathrm{dBm}$

Receiver sensitivity: $-38.0 \mathrm{dBm}$

Fiber attenuation@ $850 \mathrm{~nm}: 4.0 \mathrm{~dB} / \mathrm{km}$

Distance: $1.5 \mathrm{~km}$

8 connectors@ $0.5 \mathrm{~dB}$ each

Required loss margin: $6 \mathrm{~dB}$

Transmitter power:

$-18.5 \mathrm{dBm}$

Receiver sensitivity:

$$
\frac{-(-38.0 \mathrm{dBm})}{19.5 \mathrm{~dB}}
$$

Power budget $=$

\begin{tabular}{lr}
$4.0 \mathrm{~dB} / \mathrm{km} \times 1.5 \mathrm{~km}:$ & $6.0 \mathrm{~dB}$ \\
$8 \times 0.5 \mathrm{~dB}:$ & $4.0 \mathrm{~dB}$ \\
\multicolumn{1}{c}{ Loss margin: } & $+6.0 \mathrm{~dB}$ \\
\hline \multicolumn{1}{c}{ Total loss $=$} & $\mathbf{1 6 . 0 \mathrm { dB }}$
\end{tabular}

Since the total loss does not exceed the power budget (there are $3.5 \mathrm{~dB}$ to spare), this system provides sufficient power to the receiver for proper operation. If the required loss margin was increased to $10 \mathrm{~dB}$ (e.g., to allow for more future splices or connectors), the total loss would exceed the power budget. In this case, a more powerful transmitter or more sensitive receiver would have to be used.

The choice of operating wavelength is another important issue. The design example above was done at $850 \mathrm{~nm}$, one of three operating wavelengths used for glass fibers. For distances of a few kilometers or more, losses associated with the fiber could be appreciably reduced by using 1300 $\mathrm{nm}$ components. At $1300 \mathrm{~nm}$, fiber loss is about $1 \mathrm{~dB} / \mathrm{km}$, a reduction of about 3 or $4 \mathrm{~dB} / \mathrm{km}$ over $850-\mathrm{nm}$ operation. For short distances, this fiber loss reduction does not yield much advantage, and the designer would probably use 850$\mathrm{nm}$ components to reduce costs.

For extremely low fiber loss, 1550 -nm systems are available, but these systems are targeted for very high-speed, long-distance communication links not likely to be required in safeguards applications. These $1550-\mathrm{nm}$ systems are also very expensive.

For very short applications, plastic fiber systems present an attractive alternative. These systems are operated at $665 \mathrm{~nm}$. Plastic fiber's loss is over $100 \mathrm{~dB} / \mathrm{km}$, so its use is limited to applications of 100 meters or less. One advantage is plastic fiber's efficient coupling of transmitted light, due to its large core diameter. Plastic fiber systems (transmitters and receivers) are inexpensive compared with their glass counterparts.

Bandwidth - which is related to how much information can be transmitted down the fiber at one time-is another issue that should be addressed in the system design. Determining the fiber's bandwidth is simple: divide the fiber's frequency-length product (in $\mathrm{MHz}-\mathrm{km}$ ) by the length to be used (in $\mathrm{km}$ ). The result is the bandwidth (in $\mathrm{MHz}$ ) of that particular piece of fiber. Determining the system's bandwidth is more difficult and is beyond the scope of this report. It involves knowing the response times of the transmitter and receiver as well as the fiber's response time. Refer to Denny, ${ }_{1}^{1}$ Hecht, ${ }^{2}$ or Palais, ${ }^{4}$ for an in-depth discussion of system bandwidth, or consult with one of the many manufacturers of fiber-optic communications components.

\subsection{Installation}

Cable installation and fiber termination (installing connectors) is beyond the scope of this report. Refer to the various cable and connector manufacturers for this kind of information. (See Appendix D.)

With regard to the physical installation of a fiber-optic communications system, several practical tips are worth mentioning. ${ }^{1}$

(1) Do not mix fiber sizes or types.

(2) Plan for at least four connections for each link (one connection on each end and two intermediate connections) to allow for flexibility and future expansion.

(3) Try not to mix connector types or splice types (reduces tools and inventory).

(4) Include extra fibers for future growth.

(5) Include extra cable length to allow for future splicing when determining cable lengths.

(6) Try to place splice points inside buildings (for more convenient installation and maintenance of splices).

Some safety issues to remember include the following:

(1) Use eye protection when working with bare fiber.

(2) Collect fiber remnants on sticky tape, and seal in a closed container (e.g., a plastic bag) for safe disposal.

(3) Always assume that the fiber you are working on is illuminated by a laser diode (eye-safety standards apply), unless you know for certain that it is not. 


\subsection{Test}

Testing a fiber-optic intrusion detection system to verify proper operation can be as simple as performing intrusion trials. Testing can also be as complex as determining the exact location of a break in a fiber-optic cable. To determine the actual optical performance of a fiber-optic system, special test equipment is needed. Fiber-optic test equipment consists of optical power meters, stabilized light sources, and optical time domain reflectometers. 5

An optical power meter is a fundamental piece of fiberoptic test equipment used to measure optical power. It can also be used to determine optical power loss over a fiberoptic path. This is done by measuring the actual optical power on each side of the component being characterized (connector, splice, piece of fiber, or combination), and comparing the two measurements. This is useful for verifying the power budget of an installed system. Optical power meters can be wavelength-specific (e.g., 850-nm operation only), but multi-wavelength meters are also available that operate in all three transmission windows $(850,1300$, and $1550 \mathrm{~nm})$.

Stabilized light sources provide a known power level and wavelength of light into a fiber-optic system. These light sources are used in conjunction with optical power meters to make loss measurements when a system light source is not available. Laser diode and LED stabilized light sources are available. Test sets that incorporate both stabilized light sources and optical power meters are called optical loss test sets.

Optical time domain reflectometers (OTDRs) are used to measure fiber loss over distance and also to measure splice and connector losses in the system. In fact, a full system characterization can be made with an OTDR. Measurements are made from one end of the fiber, which is more convenient than the two-end measurement method required by optical loss test sets. Fiber failures (breaks) can be located along the fiber length with OTDRs and also with specialized OTDRs called fault locators.

\subsection{Maintenance}

Fiber-optic systems are relatively maintenance free. End-toend loss measurements are periodically recommended for fiber-optic communications systems to ensure that the systems continue to meet the design specification for power budget.

Connectors require special attention-they should always be covered with their dust caps when not in use. Also, unmated connectors should always be cleaned with a cotton swab and alcohol prior to remating.

\subsection{References}

1. Denny, J. E., Fiber Optic Cookbook for LAN and Data Link Applications, AN-007LG, AT\&T Technologies, Inc., 1986.

2. Hecht, Jeff, Understanding Fî́ber Optics, Howard W. Sams and Company, Indianapolis, Indiana, 1987.

3. Nellist, John G., Understanding Telecommunications and Lightwave Systems: An Entry-Level Guide, IEEE Press, New York, 1992, pp. 6, 105-173.

4. Palais, Joseph C., Fiber Optic Communications, Prentice-Hall, Inc., Englewood Cliffs, New Jersey, 1984.

5. Horwitz, Dennis, "How to Select Fiber-Optic Test Equipment," Communications Technology, January 1993. Also published as RIFOCS Corporation application note $\mathrm{AN}-101$. 


\section{Applications}

With respect to physical protection, fiber optics applies to two areas:

(1) communications (e.g., video, data, voice), including the protection of the optical fiber from tampering, and

(2) intrusion detection.

Another fiber-optics application area that relates to safeguards is tamper indication, although this falls under the topic of material control and accounting, and not under physical protection.

Uses of fiber optics in communications, intrusion detection, and tamper indication are discussed in the following sections.

\subsection{Communications}

Communications, as it relates to the physical protection of nuclear materials and facilities, can be divided into three categories - the transmission of video, data, or voice information. Optical fiber transmission systems are applicable to all three types of information.

There are several advantages to using fiber optics instead of metallic cables in communications. ${ }^{1}$ One advantage is that signals can be transmitted longer distances without amplifiers or repeaters. For example, a coax system carrying video signals typically incorporates a line amplifier every 1000 feet to overcome losses in the cable. On the other hand, some fiber systems can transmit study-quality video signals 30 miles without amplification.

Another advantage is the inherent security of optical fiber. It is virtually impossible for someone to passively "listen in" on the signal (eavesdrop) - the light is confined inside the fiber. This is not the case for metallic cables: an electromagnetic field is present around a metallic cable and is detectable by a knowledgeable and properly equipped individual. To counter adversary attempts to gain access to fiber-optic signals, the signal power can be monitored for appreciable change at the optical receiver. This change would indicate that an intruder was accessing the fiber.

A third benefit is fiber-optics' immunity to electromagnetic interference (EMI). EMI is commonly present at electric utility facilities and nuclear power plants and can couple into traditional communication cables, causing signal distortion and noise. Optical fiber is not susceptible to these problems and operates well in EMI-noisy environments.
Fiber-optic systems also have superior signal quality. Since fiber-optic systems are light-based, ground loop problems are eliminated, contributing to cleaner signals. Impedance variations that reduce signal quality in metallic cable systems are nonexistent in fiber. Fiber's wide bandwidth (several hundred $\mathrm{MHz}$ ) also contributes to its excellent signal quality.

Finally, fiber systems are flexible and upgradable. A security system may be required to handle video, control, and audio information. Fiber provides the flexibility to handle all these information types, and all of the signals may be multiplexed on a single fiber. Follow-on improvements to handle higher bandwidths can usually be accomplished by using the existing fiber and upgrading only the optical transmitters and receivers of the system.

Basically, any electrical signal that can be sent via wire can alternatively be converted to an optical signal and transmitted along a fiber (see Figure 5-1).

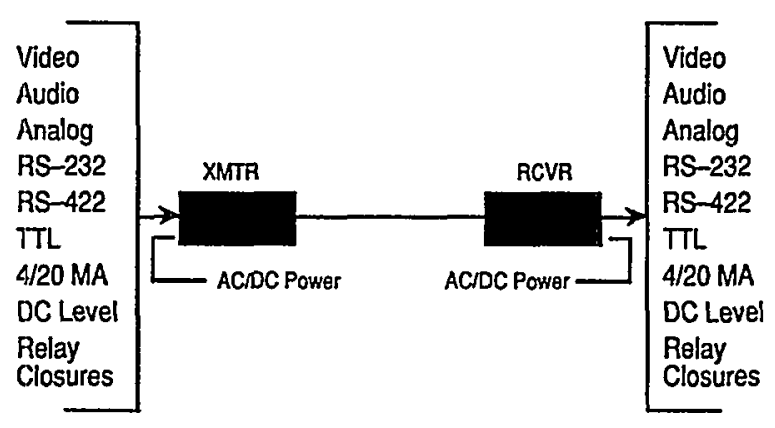

Figure 5-1. Fiber-optic transmission systems

From Math Associates, Inc., fiber optics product catalog, OSeptember 1990. Used by permission of Math Associates, Inc.

There are certainly communications applications where metallic systems are more appropriate (e.g., short distances, no EMI, low bandwidth signals)-optical fiber is no panacea. Also, there are unique installation and repair issues associated with optical fiber. During optical fiber termination operations, care should be taken to keep the fiber clean. Even small particles of dust can significantly reduce performance. Optical fiber repair (splicing) requires special tools to guarantee proper alignment of the hair-like fiber. 


\subsubsection{Video ${ }^{2}$}

Within the context of physical protection systems, video information typically originates at an assessment camera and is transmitted through a coax cable to an alarm monitoring station. There, it is displayed on a television monitor. This describes a simple closed-circuit television (CCTV) system. See Figure 5-2.

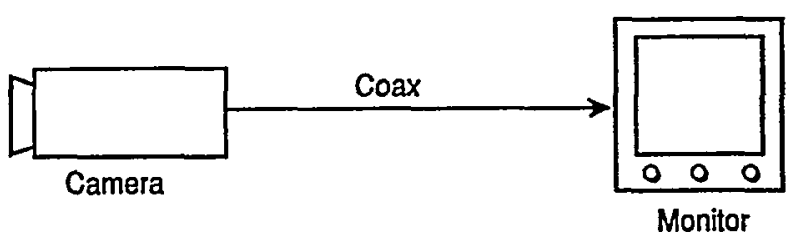

Figure 5-2. CCTV alarm assessment system using coax cable

How is CCTV accomplished with fiber optics? The coax cable is simply replaced with three components: a fiberoptic transmitter (light source), a piece of optical fiber, and a fiber-optic receiver (light detector). See Figure 5-3.

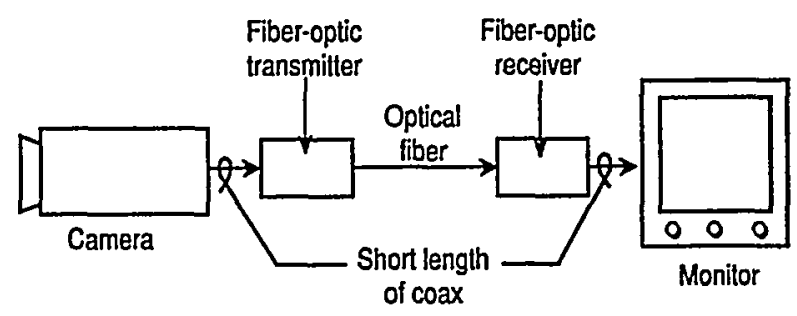

Figure 5-3. CCTV alarm assessment system using fiber optics

In this system, only the video signal is being transmitted on the fiber, the direction of transmission being from camera to monitor.

At first glance, the fiber-optic solution looks more complex than the coax system (three components versus one). However, coax systems, especially those used outdoors or those used for long runs, generally include additional components for lightning protection, signal equalization, and ground loop elimination. These additional components are not used in fiber-optic systems.

If control information (e.g., pan-tilt-zoom, or PTZ) needs to be passed from the monitoring location to the camera, another fiber (and associated fiber-optic transmitter and receiver) can be added between the monitoring station and the camera. In this two-fiber system, information travels in a single direction on each fiber-video information from camera to monitor and data (control) information from the alarm monitoring station to the camera. Fiber-optic transmitters and receivers are available that interface to a variety of PTZ signal formats, including RS-232, RS-422, Manchester (or biphase), transistor-transistor logic (TTL), or relay. Figure 5-4 shows a video system incorporating both video and control signals via optical fiber.

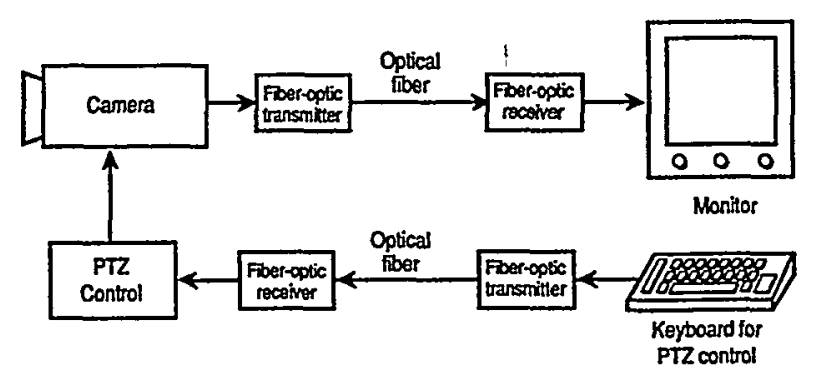

Figure 5-4. CCTV system incorporating video and control signals

Video and control signals often interface to common transceiver units. The transceiver on the camera end handles outgoing video and incoming data (transmit video/ receive data); the transceiver at the monitoring station takes care of incoming video and outgoing data (receive video/ transmit data). These transceivers could interface to two fibers as described above (one fiber for each direction) or, in more complex systems, could connect to a single fiber. Bidirectional traffic on a single fiber is handled through the use of wavelength division multiplexing (WDM), meaning that one direction uses $850-\mathrm{nm}$ components and the other direction uses 1300-nm components. Figure 5-5 illustrates this concept.

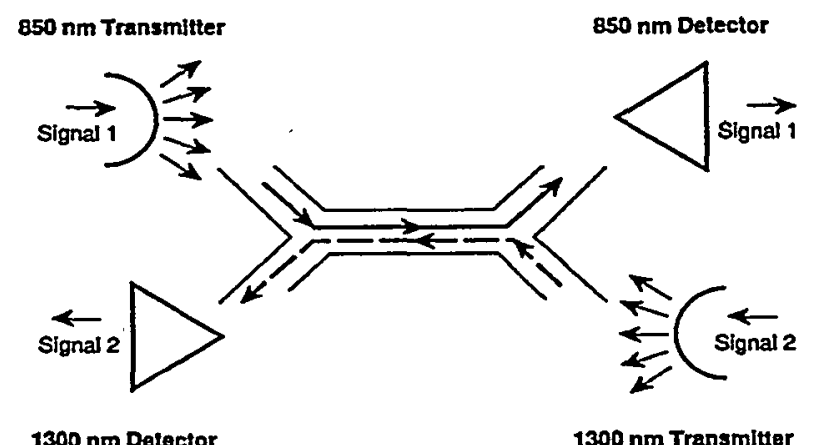

Figure 5-5. Wavelength division multiplexing

From International Fiber Systems, Inc., fiber optic data and video products catalog, 01993 . Used by permission of International Fiber Systems, Inc. 
The advantages of using fiber over copper for video transmission include: ${ }^{3}$

(1) immunity from induced noise

(2) no radiated EMI

(3) immunity from external EMI

(4) no ground-loop electrical path and associated $60-\mathrm{Hz}$ interference

(5) reduced susceptibility to equipment damage from lightning

(6) wider bandwidth capability

In cases where a high-resolution graphics terminal is remotely located from the main computer, fiber optics presents a good solution. High-resolution video signals can extend well above the $10-\mathrm{MHz}$ range present in standard video. These higher frequencies, coupled with long distances, dictate that fiber optics be used for high-quality image reproduction.

\subsubsection{Data}

The previous section (video) introduced the use of fiber optics for transmitting one kind of data: camera control information. Alarm information is another form of data commonly transmitted within physical protection systems.

A typical alarm communications system consists of sensors, multiplexers (or transponders), multiplexer control unit (MCU), and a host computer (see Figure 5-6).

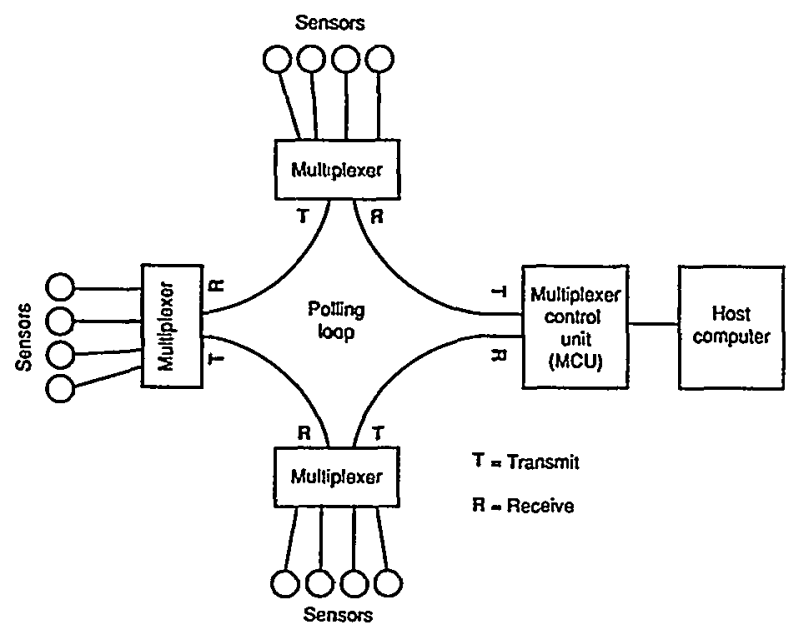

Figure 5-6. Alarm communications system

The sensors are connected to the multiplexers and provide a relay closure to the multiplexers to indicate an alarm. The multiplexers are linked to each other and the MCU by a polling loop. The MCU periodically polls (interrogates) the multiplexers; the states of the sensors are passed to the host computer for further actions (audible alarms, camera switching, map display updates, etc.).

This system has several possibilities for applying fiber optics. The most obvious application is to use optical fiber as part of the polling loop. For example, multiplexers may be separated from each other or from the MCU by large distances. Fiber can be used to span these large distances, enabling a physically larger loop than would be possible with common electrical serial interfaces such as RS-422 (maximum length: $4000 \mathrm{ft}$ ), RS-485 (4000 ft), or RS-232 $(50 \mathrm{ft})$. Fiber-optic data transmitters and receivers that interface to these serial interfaces are readily available. The fiber transmitters, receivers, and cable simply replace the copper cable.

Another possibility is the case where a sensor is distant from the multiplexer. The relay closure information ("on" or "off") from the sensor can be communicated easily via fiber to the multiplexer. Several manufacturers make fiber-optic transmitters that accept relay closures as inputs. Fiber-optic receivers are available that provide relay closures as outputs.

Many alarm communications systems, like the one shown in Figure 5-6, have redundancy built in so that failures of the MCU, host computer, or polling loop do not cause the whole system to fail. The backup system that provides this redundancy would probably be located at another site (an alternate alarm monitoring station). Communication between the primary and backup systems provides a good opportunity to use fiber optics. Linking the two systems is readily done via a fiber-optic local area network (LAN). Products to accomplish a fiber-optic LAN implementation are available from a number of manufacturers.

Frequently, physical protection systems permit authorized personnel to enter protected areas through the use of automated entry control equipment-personal identification numbers (PINs), card readers, hand geometry readers, etc. Communication between the remotely located entry control equipment and the alarm monitoring station is another application for fiber optics.

\subsubsection{Voice}

Transmission of voice signals via optical fiber brings to mind the widespread use of fiber optics in the long-distance telephone industry. Although a fiber-optic telephone system is most likely not required for a physical protection system, a fiber-optic intercom (audio) system is easily achievable with products now available. Figure 5-7 illustrates an 
example of a fiber-optic video-audio link that might be used in an entry control booth of a physical security system.

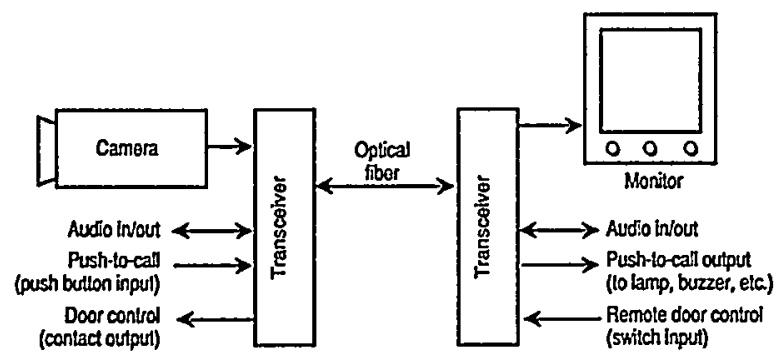

Figure 5-7. Video/audio link using fiber optics

In addition to video and audio, push-to-call and remote contact closure (e.g., to operate a door lock) functions can be provided over the same link.

\subsubsection{Communications Products}

A variety of fiber-optics products exists that supports transmission of video, data, and voice signals. A partial list of fiber-optics equipment suppliers is included as Appendix D.

\subsection{Intrusion Detection}

Fiber-optic intrusion sensors detect (1) cutting or breaking of the fiber or (2) light disturbances. ${ }^{4}$ These sensors can be:

(1) installed on fences

(2) buried in the ground

(3) placed under carpet or above ceiling tiles

(4) built into grates, grids, and window screens

(5) embedded into walls

Sensors that detect only the cutting or breaking of the fibers do so by monitoring the optical continuity of the fiber. A light source sends a signal through the fiber to the detector. As long as the signal arrives at the detector, no alarm occurs. If the light is interrupted by a cut or break, an alarm is reported (see Figure 5-8).

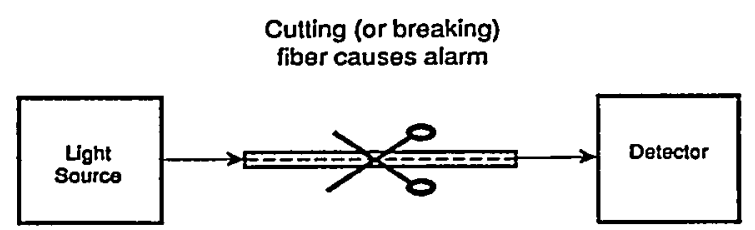

Figure 5-8. Causing an alarm on an optical continuity sensor
Optical continuity sensors have a low nuisance-alarm rate since the light path must be positively broken to be considered an alarm.

Light disturbance sensors detect minute microbending of the fiber caused by environmental changes such as pressure, motion, or vibration (see Figure 5-9).

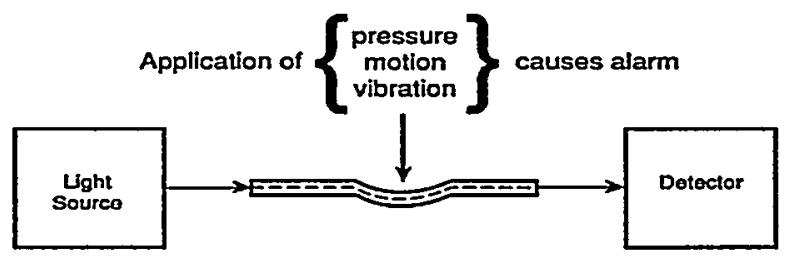

Figure 5-9. Alarm causes for a light disturbance sensor

Light disturbance systems include processing circuitry to determine if the environmental change is sufficient to declare an alarm. For this reason, these sensor systems are significantly more complex than optical continuity sensors. The sensitivity (to environmental change) of most light disturbance systems can be adjusted to optimize the detection probability and nuisance-alarm rate for a particular installation.

Fiber-optic intrusion detection systems are relatively new, having been introduced to the physical protection market only in the last few years. Consequently, their reliability and maintainability are not as well known as that of more established sensor technologies such as microwave and infrared systems.

The following subsections describe intrusion detection products. Light disturbance products are discussed first, followed by a description of optical continuity products.

\subsubsection{Light Disturbance Products}

Several commercial products are available that detect light disturbances of optical fiber due to pressure, motion, or vibration. These products can be configured for a variety of applications. They can be:

(1) buried in the ground

(2) attached to mechanical or structural fixtures (fences, walls, etc.)

(3) embedded in walls 
(4) placed under carpet or above ceiling tiles

(5) placed under articles to be protected

Common configurations include burying the sensing cable or attaching it to a fence. In buried applications, pressure changes caused by an intruder moving over the sensored area are transferred to the fiber cable and detected. In fencemounted applications, fence vibration and movement caused by cutting, climbing, bumping, or lifting of the fence fabric are induced in the fiber cable and detected.

Techniques for detecting light disturbance differ somewhat for each manufacturer. All of the systems consist of an optically sensitive fiber cable (deployed in the desired environment), a light source, a detector, and alarm processing circuitry (see Figure 5-10).

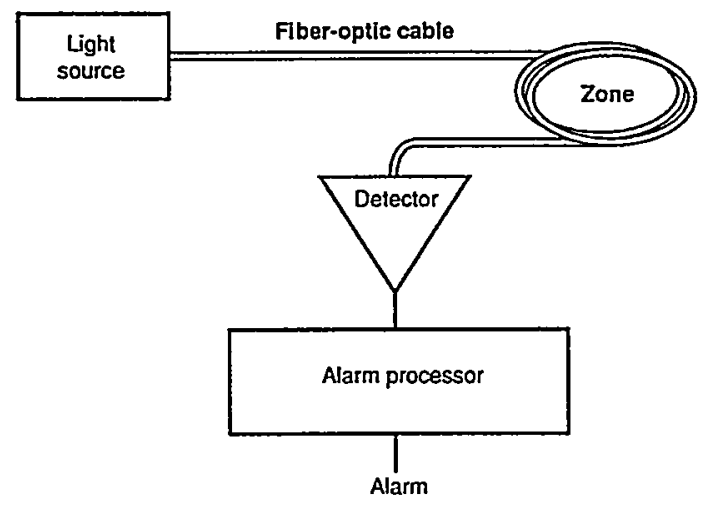

Figure 5-10. Typical components of a fiber-optic light disturbance sensor

From Fiber SenSys, Inc., Fiber Optic Intrusion Detection System Seminar Manual. Used by permission of Fiber SenSys, Inc.

The light source, detector, and alarm processor are frequently contained in one unit. Although manufacturers are willing to discuss general concepts of operation, details of the actual detection technique are generally proprietary.

One type of fiber-optic intrusion detection system uses a multimode interferometric technique. ${ }^{5-7}$ In addition to a multimode interferometer, the system consists of a laser diode emitter, a sensitive multimode fiber, a light detector, and qualification processing. A system using these components requires the detection equipment to be located at the zone of detection. Otherwise, movement of the sensitive cable leading from the intended detection zone to the detection equipment would produce undesired alarms. For installations in which the secure zone is remote from the detection equipment, insensitive fiber-optic lead-in cable is available.

The detection method involves monitoring mode interference changes of the light (up to 60 modes may be propagated in the fiber) due to pressure, vibration, or motion. To optimize detection capability and minimize invalid alarms for a particular installation, the system allows the user to select appropriate processing parameters to qualify a disturbance as an alarm. These parameters include the frequency band, energy level and duration of the disturbance, and the number of disturbances within a specified time. An alarm is indicated by a relay closure, an indicator lamp, and an audible signal. The system can be reset automatically or manually.

One group of fiber-optic intrusion detection systems uses an optical interferometry technique in which laser beams are transmitted into each end of a single-mode fiber.8,9 The interaction between the two beams yields a high sensitivity to disturbances of the fiber. Pressure (movement, sound, vibration) on the fiber changes the relative phases of the beams. This phase change is detected and processed for alarm qualification. In some of the products, the user may optimize the system to discriminate between environmental and intruder disturbances by appropriately selecting the sensitivity, detection threshold, time window, event count, and frequency window. Other products allow user adjustment of the sensitivity only. The use of single-mode fiber allows sensor loops of more than $30 \mathrm{~km}$ without the aid of amplifiers or repeaters. These systems are advertised for use in both indoor and outdoor applications and may be buried in the ground (see Figure 5-11, next page); mounted on fences, walls, towers, or other structures; attached to or placed beneath artifacts; or used as acoustic sensors to monitor sound.

Another group of fiber-optic intrusion detection sensors also uses a laser. ${ }^{10-11}$ Laser light transmitted into a multimode fiber causes modal interference and forms a speckle pattern at the detector end of the fiber (telephone interview with Douglas LeBarge, Stellar Systems, October 7, 1993). Mechanical disturbances to the fiber (pressure, vibration, motion) cause a change in the speckle pattern that is detected and processed for alarm qualification. Alarm threshold and laser power output are user-adjustable to optimize performance for a particular installation. Two of these light disturbance systems are available: one for buried installations (in gravel or under sod) and one for fence mounting. 


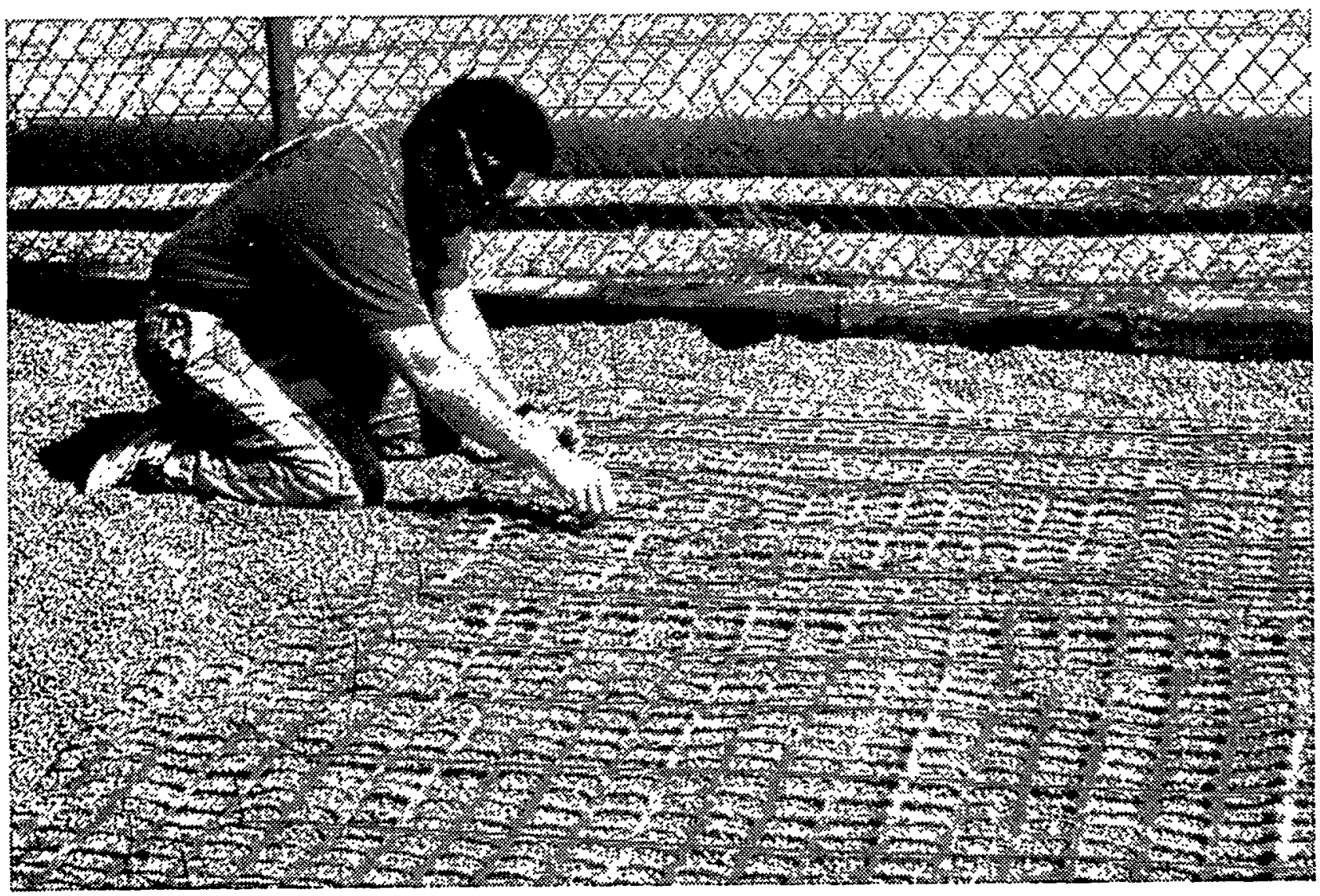

Figure 5-11. Buried installation of fiber-optic intrusion detection system

Several of the above systems have been evaluated for use in buried and fence-mounted applications. ${ }^{*} 12$ The environment of the site and the method of installing the fiber-optic system influence its performance. Cold weather is a concern for the buried fiber-optic systems since frozen ground desensitizes these systems.

Other fiber-optic sensors include a decorative fence and a submergible grid barrier, both of which utilize fiber-optic light disturbance to detect intrusions. ${ }^{13-16}$ In the decorative fence, a fiber-optic sensing cable is installed in the top vertical support channel of the fence. An emitter at one end of the cable transmits a light pulse into the fiber; this light pulse is detected at the other end. Mechanical pressure caused by attempts to climb or bend the fence structure are transferred to the fiber via a sensing device inside the fence. The disturbance of the fiber attenuates the light signal arriving at the detector and an alarm is initiated. Attempts to cut the fence structure are also detected. The submergible

\footnotetext{
'Test Report: Metricor Fiber-Optic Intrusion Detection System, EG\&G 851, Communications Security Design, November 1990. (proprietary report, not publicly available).
}

grid serves as a physical barrier for water flow openings. Its construction and detection mechanism are similar to the decorative fence. Attempts to bend, spread, or cut the structure result in a reduction of the light signal arriving at the detector and consequently initiate an alarm. The intrusion detection method used in both the decorative fence and the submergible grid is classified as optomechanical.

Another fiber-optic intrusion detection system under development is expected to be able to both locate the intruder (along the perimeter) and classify the class of intrusion (crawler, walker, runner, vehicle). ${ }^{17}$ Buried, ground surface, and fence-mounted installations will be permitted. Instead of an optical loop, the system uses an optical fiber with both the emitter and detector located at the near end; the far end is mirrored. Collocating the emitter and detector requires optical couplers to interface these components to the fiber. The system uses a laser source and multimode fiber and is sensitive to phase interference due to the application of pressure to the fiber. A working model of the system has been demonstrated. 


\subsubsection{Optical Continuity Products}

Several fiber-optic intrusion detection products are available that detect loss of optical continuity (i.e., a break in the fiber). These sensors can be:

(1) built into barbed-tape barriers

(2) built into water inlet and outlet meshing

(3) embedded in walls

(4) built into window screens

Optical continuity systems consist of an emitter, a detector, and the optical fiber. Light signals originating at the emitter pass through the fiber and are received at the detector. Receipt of these signals at the detector confirms the integrity of the fiber. Loss of the light signal resulting from cutting or breaking of the fiber indicates an intrusion.

Optical continuity intrusion detection systems include fence and web-type sensors (telephone interview with Douglas LeBarge, Stellar Systems, October 7, 1993).10-11 In one product, an optical fiber is encased in steel barbed tape. This barbed tape can serve as a stand-alone fence (providing a physical barrier) or can be fitted to walls, existing fences, or gates. Another product consists of a mesh or web of fiber cable that resembles rigid chain-link fencing. It can be used by itself to provide a sensitive physical barrier or can be incorporated into structures (walls, ceilings, floors). Attempts to penetrate the structure (or the mesh itself) will break the fiber and generate an alarm. A similar mesh product is submergible and can provide intrusion protection of water inlets and outlets.

A fiber-optic window screen is also available that utilizes the optical continuity concept. 9 Optical fibers woven into the screen allow the system to detect cutting of the screen. Recommended installations include safe rooms, high-ticket manufacturing plants, and museums.

Fiber-optic continuity proof-of-concept systems have also been demonstrated. One system, developed for treaty verification applications, consists of a fiber-optic cable threaded through a fence. ${ }^{18}$ The system monitors the integrity of the fence via optical continuity of the fiber and is used to indicate large-scale breaches of the fence (e.g., cable cuts and fence dismantlements for vehicle or treatyitem passage). The system also employs techniques to detect fiber-optic tampering. In addition to fence installations, the fiber-optic cable could also be installed on walls or secure containers.

\subsection{Tamper-Indicating Devices (Loop Systems)}

Several fiber-optic systems are available that provide tamper indication. These systems are used to show either unauthorized access or attempts to steal assets or equipment. All fiber-optic tamper-indicating systems use a loop of optic fiber cable that is threaded through or around an object. If the cable is broken or cut, light transmitted in one end will not be detected at the other end, indicating removal of the seal and possible item tampering. Some systems (seals) are passive-light is passed through the fiber during installation or verification operations only. Other systems (e.g., anti-theft products) are active-light signals are continuously transmitted through the loop. The following two subsections describe passive and active tamper indicators.

\subsubsection{Passive Tamper Indicators (Seals)}

These systems include two treaty verification seals and a commercial seal.

The first system is used to seal items under the control of nuclear nonproliferation treaty agreements (interview with Ken Ystesund, Sandia National Laboratories, July 1993). ${ }^{19,20}$ The system consists of a length of multi-strand fiber-optic cable (containing 64 plastic fibers) and a clear polycarbonate seal body (to secure the ends of the cable). See Figure 5-12.

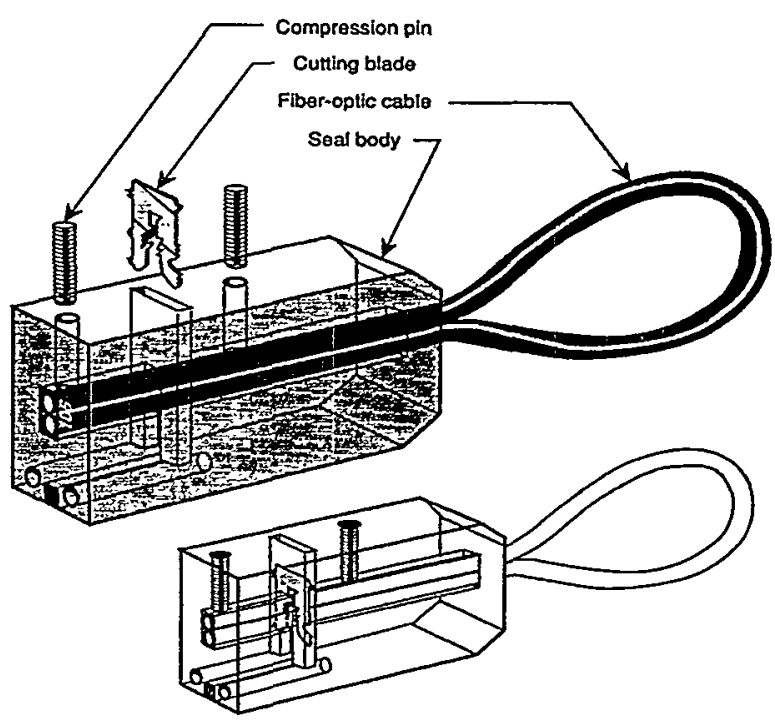

Figure 5-12. Treaty verification seal 
The use of plastic fibers allows the cable to be sheared off cleanly without the use of special tools.

The seal is used as follows. A fiber-optic cable (up to $20 \mathrm{~m}$ ) is threaded through or around the item to be sealed. The two ends of the cable are then inserted into the seal body, which is pre-loaded with a cutting blade. An installation tool compresses the seal body and pushes the cutting blade into the cable. Due to the construction of the cutting blade, some of the fibers are cut and some are not. The random construction and placement of the cable give the seal a unique signature. Next, the cable ends are cut at the seal face with a cutting tool. Light is shone into the fiber bundles, and an end-view image of the fiber bundles and seal body is recorded (Polaroid-type photograph). Broken fibers do not transmit light and therefore appear dark; unbroken fibers appear bright. To verify seal integrity, a picture is taken of the seal body. This new image is compared with a previous image (made when the seal was installed) to determine whether the fiber loop has been broken or replaced. A proof-of-concept electronic seal verifier has been demonstrated that performs an automated comparison of images. ${ }^{21}$

The second system is an improvement of the first seal and is also used for treaty verification (interview with Jack Bartberger, Sandia National Laboratories, July 1993). The system has a more secure seal body and a different cable jacketing that includes unique identifiers (cable length codes and multi-colored particles embedded in the clear jacketing). It is under development at Sandia National Laboratories with DOE funding.

The commercial seal was developed for use by the public utility, transportation, and security industries. ${ }^{22}$ The seal consists of two basic parts: a high-impact plastic body and a fiber-optic cable. See Figure 5-13.

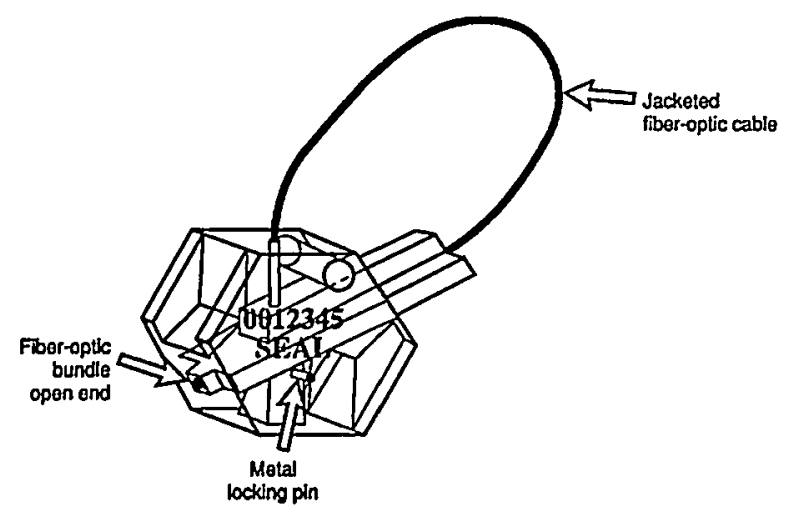

Figure 5-13. Commercial seal
The seal body is heat-stamped with a serial number and optional company name, and securely holds the ends of the fiber cable. The cable consists of randomly positioned acrylic optical fibers covered with a protective polyethylene jacket. Cable loops less than 50 feet have 16 fibers; longer loops have 24 fibers. Seal integrity can be checked by simply shining a light in one end of the cable and observing whether light is emitted from the other end. Alternatively, a handheld device is available that provides an audible indication of the seal's integrity. If documented evidence of the seal status is required, a camera verifier is available.

\subsubsection{Active Tamper Indicators}

Active tamper indicators include a couple of anti-theft products and a proof-of-concept fiber-optic necklace (used in personnel tracking). The two anti-theft systems are intended to secure equipment highly susceptible to theft (e.g., personal computers and peripherals). In these systems, a fiber-optic cable is threaded through the equipment that requires protection. The cable connects to a box containing the emitter, detector, and control circuitry. An alarm is initiated if the cable is cut, broken, or disconnected (telephone interview with Bob Putrino, Photonic Alarm Products, September 1993).23

A proof-of-concept fiber-optic necklace has been demonstrated as part of a personnel and material tracking system. ${ }^{24,25}$ The necklace fastens an RF tracking tag to a person and will not allow unauthorized removal. Attempts to cut, break, or remove the fiber cable are detected, initiating an alarm.

\subsection{References}

1. Cladis, Chris P. and Mitch Frishman, "The Force of Fiber," Security Management, October 1990, pp. 7780.

2. De Lia, Robert, "Seeing into the World of Fiber Optics for Security," Security Management, March 1993, pp. 7A-11A.

3. Greenwoll, D. A., J. C. Matter, and P. E. Ebel, Video Systems for Alarm Assessment, NUREG/CR-5721 (SAND91-0947), September 1991.

4. Vitch, Martin L., "Sensing Your Way to Security," Security Management, July 1992, p. 54.

5. Reynolds, Sandra, "Fiber Optic Intrusion Detection," Proceedings of the 6th Annual Joint GovernmentIndustry Symposium and Exhibition on Security Technology, June 12-14, 1990, Williamsburg, Virginia (American Defense Preparedness Association). 
Applications

6. Fiber SenSys, Inc., Model 105E/106E Fiber Optic Intrusion Detection Systems Installer's Manual.

7. Reynolds, Sandra, "Fiber Optic Sensors Offer Various Options," Access Control, March 1991.

8. Mason and Hanger National, Inc., FOIDSTM: Fiber Optic Intelligence \& Detection System, sales brochure literature.

9. "Indoor Fiber: Built-in Wall, Window Security," Security, May 1993, p. 59.

10. Remsdaq Ltd., Sabre Perimeter Systems, product literature.

11. Stellar Systems, Fibre Optic Sensors and Systems for Security, product literature.

12. This reference intentionally lef blank

13. Safeguards Technology, Inc., DTR DF Decorative Fence Manual.

14. Safeguards Technology, Inc., DTR-DF Decorative Fence, product literature.

15. Safeguards Technology, Inc., MAGBAR OPTO GRID Operation and Maintenance Manual.

16. Safeguards Technology, Inc., MAGBAR OPTO GRID Intrusion Detection Grid, product literature.

17. Anro Engineering, Inc., Optical FIBer Intrusion LOCation Sensor System (FIBLOC) for Surface and Subsurface Perimeter Protection: Final Report, August 31, 1993.
18. Skogmo, David, and Bill Black, "A Fiber-Optic Barrier Integrity Monitor, "Proceedings of the $31^{\text {st }}$ Annual Meeting of the Institute of Nuclear Materials Management, July 1990, pp. 745-751.

19. Ystesund, Kenneth. J., and Darryl D. Drayer, "Laboratory/Field Evaluation of Modified Cobra Seal System," Proceedings of the 30th Annual Meeting of the Institute of Nuclear Materials Management, July 1989, pp. 875879.

20. Product literature, Cobra Seal Il, Aquila Technologies Group, Inc., October 1990.

21. Mukaiyama, T. and Y. Yamamoto, "Cobra-A Demonstration of the Development of C/S Equipment at JAERI: Electronic Verifier of Cobra Seal (Abstract)," Proceedings of the $31^{\text {st }}$ Annual Meeting of the Institute of Nuclear Materials Management, July 1990, p. 822.

22. Product literature, Brooks Fiber-Lock Seal, E. J. Brooks Company, August 1992.

23. Interactive Technologies, Inc., LightGard, product literature.

24. Anspach, DeNise A., et al., "PAMTRAK: A Personnel and Material Tracking System," Proceedings of the $33^{\text {rd }}$ Annual Meeting of the Institute of Nuclear Materials Management, July 1992, p. 681.

25. Trujillo, Amado A. and Chris E. Hoover, "Demonstration of a Real Time Personnel and Material Tracking System," Proceedings of the $30^{\text {th }}$ Annual Meeting of the Institute of Nuclear Materials Management, July 1989, pp. 392-3. 


\section{Appendix A \\ Optical Fiber Theory of Operation}

\section{A.1 Refraction, Total Internal Reflection, and Numerical Aperture $^{1-3}$}

Although the wave theory of light is necessary to completely model the light properties of optical fiber, the ray optics model is sufficient for a basic understanding of fiber optics. This section uses the ray optics model to discuss the concepts of refraction, total internal reflection, and numerical aperture.

As a ray of light crosses the boundary between two transparent media (e.g., air and glass), it is refracted, or bent. The amount of refraction, or bending, depends on the index of refraction $n$ of each material and on the angle of incidence $\theta$. (For glass, $n$ is approximately 1.5 , and for air, $n=$ 1.0.) Snell's law describes this relationship as

$$
n_{0} \sin \theta_{0}=n_{1} \sin \theta_{1}
$$

where $n_{0}=$ the index of refraction of the medium the light is traveling from

$n_{1}=$ the index of refraction of the medium the light is traveling to

$\theta_{0}=$ the angle between the incident ray and the normal to the surface

$\theta_{1}=$ the angle between the refracted ray and the normal to the surface

Figure A-1 shows the case where light is passing from a material of high index of refraction into a material with a lower index of refraction.

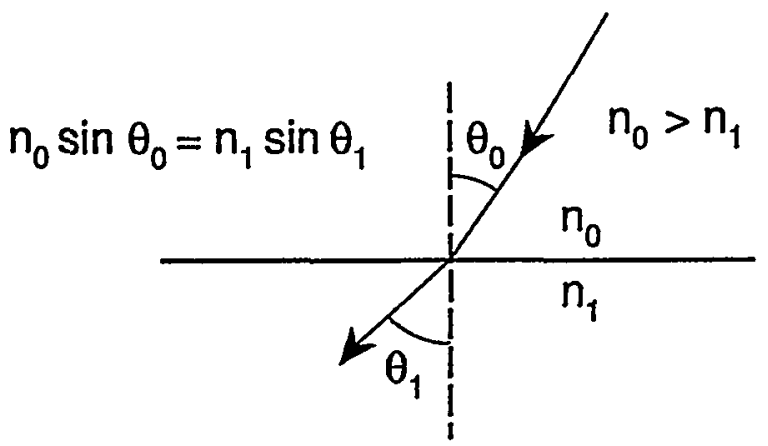

Figure A-1. Light refraction
In reality, not all of the light penetrates into the second material-some light is reflected off the boundary back into the first material.

If the angle of incidence $\left(\theta_{0}\right)$ in Figure A-1 is allowed to increase, an angle will eventually be reached where the angle of refraction $\left(\theta_{1}\right)$ is $90^{\circ}$. At this point the refracted ray follows the surface boundary. The angle at which this occurs is called the critical angle, $\theta_{c}$. The critical angle is computed as

$$
\theta_{c}=\sin ^{-1}\left(n_{1} / n_{0}\right)
$$

If the angle of incidence is allowed to increase beyond $\theta_{c}$ no light penetrates into the second material. Instead, all of the light reflects off the surface boundary. This important phenomenon, called total internal reflection, is the reason that light can be guided by an optical fiber.

A problem with using a bare optical fiber to guide light is that some light is lost wherever it is touched or supported by a non-transparent (lossy) material. The solution is to coat the fiber with a transparent medium having a refractive index less than the inner light-guiding material. Thus, optical fiber consists of a light-guiding core and a surrounding optical "insulator," called the cladding (Figure A-2). In addition to its optical benefits, the cladding physically protects the core from scratching.

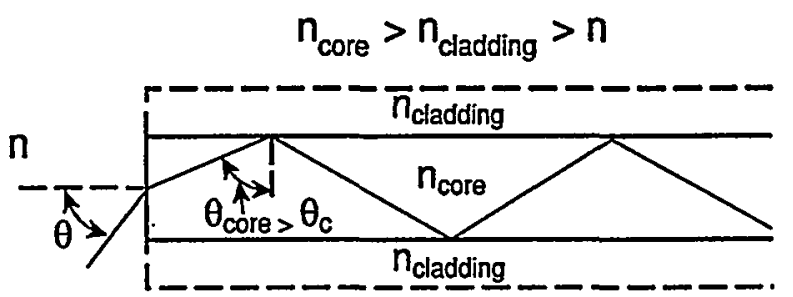

Figure A-2. Optical fiber showing the propagation of light through the core

The core has a higher index of refraction than the cladding, which permits total internal reflection if the angle of incidence is greater than the critical angle. Light can thus be confined in the core and transmitted along the length of the fiber.

Consider an external ray of light (Figure A-2). As it enters the core, the ray of light is refracted and travels to the wall 
of the core's cylinder (the core/cladding interface). If the angle of incidence at this interface is greater than $\theta_{c}$, the light will reflect off the cylinder wall, continue through the fiber (experiencing multiple reflections), and eventually emerge from the other end of the fiber. Thus, the light is guided through the core. Of course, if the angle of incidence at the core/cladding interface is less than $\theta_{c}$, light will be transmitted into the cladding and will eventually be lost at points where the cladding is touched by the non-transparent coating and buffering materials that exist in fiber cable.

One important measure of a fiber's ability to accept light is called numerical aperture, $N A$. It is dependent on the refractive indexes of the core and cladding.

$$
N A=\sqrt{n_{\text {core }}^{2}-n_{\text {cladding }}^{2}}
$$

Alternately,

$$
N A=\sin \theta_{\text {acceptance }}
$$

where $\theta_{\text {acceptance }}$ is the acceptance angle shown in Figure A3.

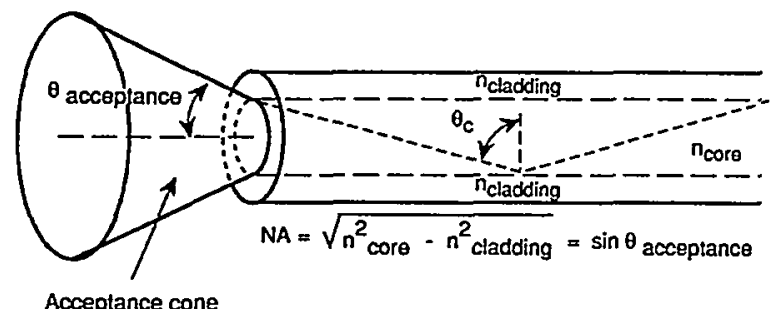

Figure A-3. Numerical aperture and acceptance angle

For angles of incidence occurring inside the acceptance cone shown in the figure, light will be propagated through the core of the fiber. In brief, the larger the numerical aperture, the larger the acceptance angle.

Optical fibers are most commonly made of fused silica. The core of the fiber is doped with germanium or phosphorus pentoxide to increase the refractive index. The cladding is either pure silica or doped with boron oxide or fluoride to lower its refractive index.

So far, we have considered only straight pieces of fiber. What happens if the fiber is curved? Figure A-4 shows a light ray traveling through the core of a curved fiber.

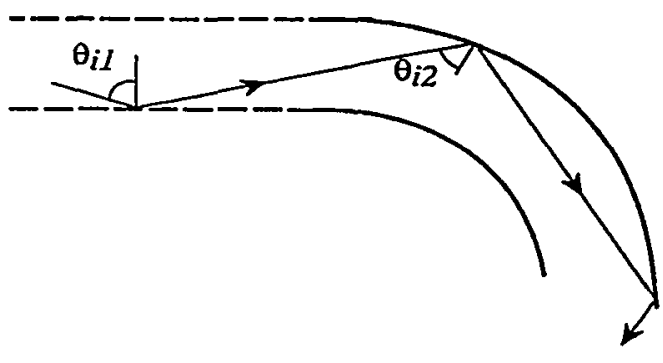

\section{Figure A-4. Effects of curved fiber on light transmission}

As long as the light ray's angle of incidence through the curved section is greater than the critical angle, all of the light will be transmitted through the curve. [Note, however, that the angle of incidence in the curved section is less than the angle of incidence in the straight section $\left(\theta_{i 2}<\theta_{i 1}\right)$, since the normal to the core surface has been rotated.] If the angle of incidence exceeds the critical angle (i.e., too tight a bend), some of the light will penetrate into the cladding and be lost. In this case, the ray would experience a loss of power (brightness) as it traveled through the curve.

\section{A.2 Attenuation ${ }^{1-3}$}

Fiber is not $100 \%$ efficient in transmitting light. Light is lost, or attenuated, as it travels through the fiber due to impurities in the fiber and imperfections in the manufacture of the fiber. Attenuation depends on the ratio of output power and input power, according to the following logarithmic relationship.

$$
\text { Attenuation }=-10 \log \left(P_{o} / P_{i}\right) \mathrm{dB}
$$

where $P_{o}=$ output power

$P_{i}=$ input power, and

the units are in $\mathrm{dB}$ (decibels).

For example, if the light's output power diminishes to 0.01 of the input power for a particular piece of fiber, the attenuation is $20 \mathrm{~dB}$. Fiber is rated according to its attenuation per unit length, usually in $\mathrm{dB} / \mathrm{km}$. For the preceding example, if the piece of fiber is $10 \mathrm{~km}$, the attenuation per unit length is $2 \mathrm{~dB} / \mathrm{km}$.

Loss is dependent on wavelength. Figure A-5 shows a typical fiber's attenuation $(\mathrm{dB} / \mathrm{km})$ over the range of wavelengths used in fiber-optic systems. This range is just beyond the visible region in the near infrared. 


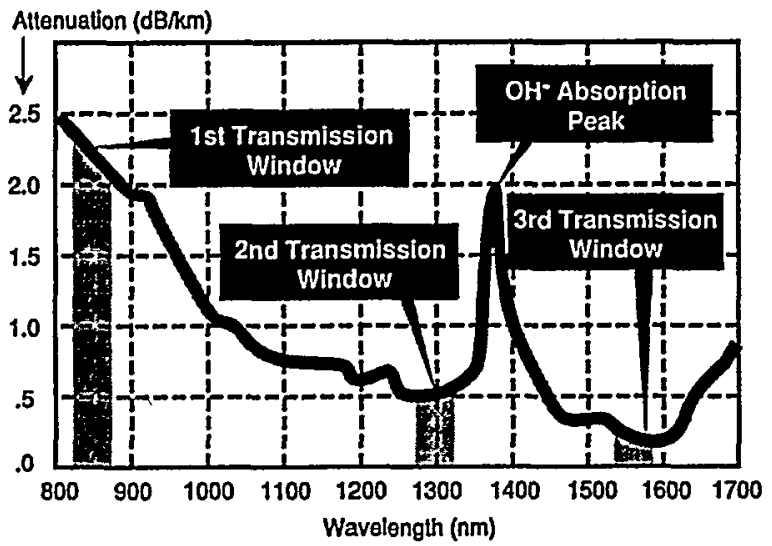

Figure A-5. Typical fiber's attenuation as a function of wavelength

From the book Understanding Telecommunications and Lighnvave Systems: An Entry-Level Guide, by John G. Nellist, O1992. Published by IEEE Press. Used by permission of publisher.

The peaks in attenuation at $1250 \mathrm{~nm}$ and $1390 \mathrm{~nm}$ are due to light absorption by hydroxyl $\left(\mathrm{OH}^{-}\right)$ions trapped in the fiber during manufacture. Premium grade fiber minimizes these impurities through an improved manufacturing process to reduce these attenuation peaks.

As shown in Figure A-5, there are three transmission "windows" used in glass fiber-optic communications$850 \mathrm{~nm}, 1300 \mathrm{~nm}$, and $1550 \mathrm{~nm}$. The last two windows were chosen as improvements to the original $850-\mathrm{nm}$ window to minimize transmission losses required for longdistance light-wave communication systems.

The general trend of Figure A-5 indicates that attenuation decreases with increasing wavelength. This is due to Rayleigh scattering, which reduces light transmission due to local (i.e., microscopic) fluctuations in density and refractive index.

\section{A.3 Dispersion Limitations of Optical Fibers ${ }^{1,2}$}

Attenuation is not the only signal-limiting characteristic of optical fiber. Modal dispersion and chromatic dispersion distort light signals by causing a spreading of the optically transmitted pulses. These concepts are described below.

Early optical fibers were "step-index" multimode fibers. In a step-index fiber, the refractive index of the core is constant from the center of the fiber to the core/cladding interface, followed by an abrupt refractive index change at the interface. Figure A-6 shows an example of the refractive index profile for a step-index multimode fiber.

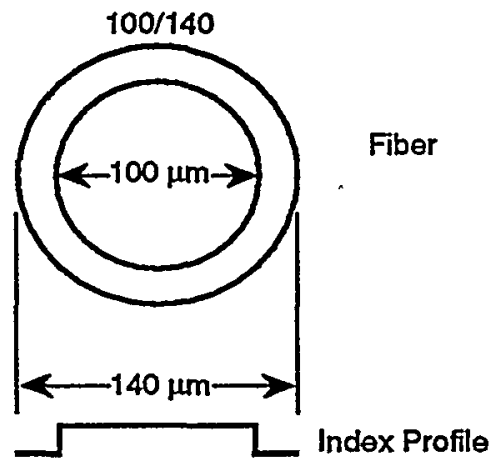

Figure A-6. Step-index multimode fiber

From the book Understanding Fiber Optics, by Jeff Hecht, 01987. Published by SAMS Publishing, a division of Prentice Hall Computer Publishing. Used by permission of publisher.

These step-index multimode fibers have a large core $(50 \mu \mathrm{m}$ or larger) and support more than one light propagation mode. The large core has the advantage of being able to collect more light than the smaller core single-mode fibers, but the disadvantage is that modal dispersion can cause signal distortion in even reasonably short lengths of stepindex multimode fiber.

As shown in Figure A-7, some of the light rays pass straight down the core, but other rays are reflected off the corel cladding interface, following a zigzag path. These zigzag rays (or light modes) travel farther and thus take longer than the straight-path ray (mode) to arrive at the end of the fiber. Since light is the summation of all of these modes, light pulses are broadened by the time they reach the output. Consequently, fiber length adversely affects the performance of step-index multimode fiber. This phenomenon is known as modal dispersion.

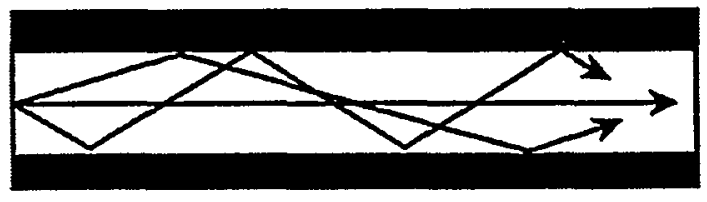

Figure A-7. Modal dispersion in step-index multimode fiber

From the book Understanding Telecommunications and Lightwave Systems: An Entry-Level Guide, by John G. Nellist, O1992. Published by IEEE Press. Used by permission of publisher.

To alleviate the pulse-broadening drawbacks of step-index multimode fiber, graded-index multimode fiber was developed. The refractive index of the core decreases parabolically from the center of the fiber to the core/ 
cladding boundary. Figure A-8 shows an example of the refractive index profile for a graded-index multimode fiber.

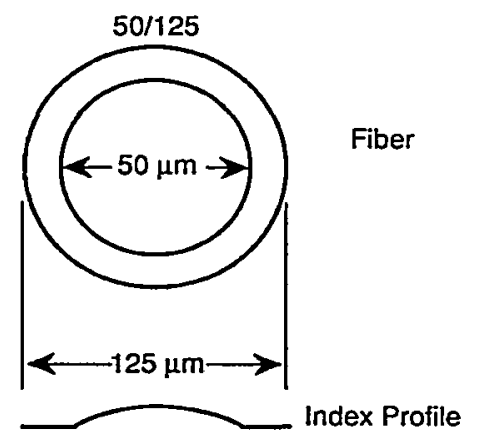

Figure A-8. Graded-index multimode fiber

From the book Understanding Fiber Optics, by Jeff Hecht, (1987. Published by SAMS Publishing, a division of Prentice Hall Computer Publishing. Used by permission of publisher.

The result of index grading is that each mode travels longitudinally along the fiber at the same rate; thus, pulses arriving at the end of the fiber are not spread out as in stepindex fiber. Each light mode travels in sine-wave-like paths through the fiber. See Figure A-9.

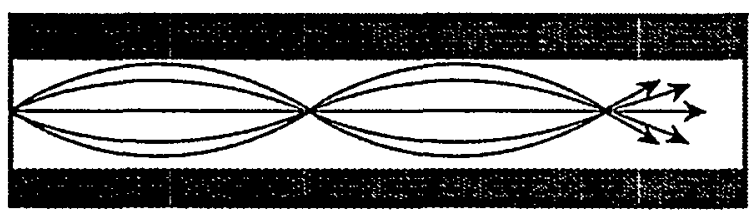

\section{Figure A-9. Correcting modal dispersion through index grading}

From the book Understanding Telecommunications and Lightwave Sensors: An Entry-Level Guide, by John G. Nellist, (1992. Published by IEEE Press. Used by permission of publisher.

In practice, some pulse broadening occurs in graded-index fiber.

One way to eliminate the adverse effects of modal dispersion is to reduce the diameter of the core to about $8 \mu \mathrm{m}$. This small diameter will support only one mode-the mode that travels straight through the fiber. Fiber that exhibits this monomode behavior is called single-mode fiber. Of course, there is no need to grade the refractive index; single-mode fiber is step-indexed. Figure A-10 shows an example of the refractive index profile for a step-index single-mode fiber.

Pulse broadening due to modal dispersion is eliminated in single-mode fiber.
Chromatic (or material) dispersion is a second pulsebroadening effect present in all optical fiber systems. Chromatic dispersion arises from the fact that (1) light sources used in fiber systems are not monochromatic, but occupy a finite spectral width (i.e., a range of wavelengths), and (2) the velocity of light through an optical material varies with the wavelength of the light.

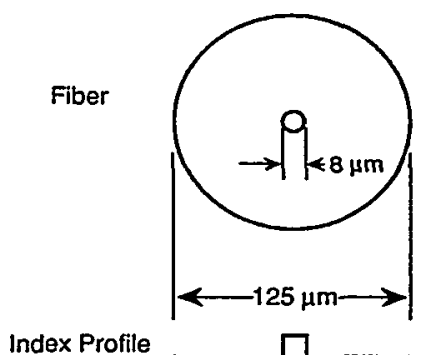

Figure A-10. Single-mode fiber

From the book Understanding Fiber Optics, by Jeff Hecht, (O1987. Published by SAMS Publishing, a division of Prentice Hall Computer Publishing. Used by permission of publisher.

A light pulse is actually the sum of many component pulses that are identical except for their wavelengths. Consider a fiber system consisting of a light source, a piece of fiber, and a detector. Imagine that the source emits a light pulse. The component pulses are emitted from the source at exactly the same time, but as they travel the length of the fiber they begin to separate in time. The component (wavelength) pulses of the original pulse arrive at the detector at different times. After a summation of the received pulses, the result is pulse spreading. By using a light source that is nearly monochromatic (e.g., a laser diode), the effects of chromatic dispersion can be minimized.

Chromatic dispersion is not constant but varies with wavelength. At approximately $1300 \mathrm{~nm}$, chromatic dispersion is at a minimum for fused silica fiber (i.e., glass fiber). By operating at this wavelength, chromatic dispersion effects can be minimized. Systems operating in the second transmission window $(1300 \mathrm{~nm}$ ) shown in Figure A-5 take advantage of this fact. Specially designed dispersion-shifted fiber is also available that has its chromatic dispersion minimum at $1550 \mathrm{~nm}$. Systems using this fiber reap the benefits of both minimum chromatic dispersion and low attenuation (recall Figure A-5).

Dispersion effects also influence the maximum light-pulse rate (bit rate) of fiber-optic systems. The basic concept is that if pulses are broadened enough, they will begin to 
"smear" into adjacent pulses. To prevent this smearing, the bit rate is reduced, effectively reducing the maximum operating frequency of the transmitted signal.

Frequency-length products (in MHz-km) are published in manufacturers' data sheets and are used to obtain maximum operating frequencies. To find out the maximum operating frequency for a given piece of fiber, divide the frequencylength product by the length of the fiber. For example, suppose the fiber is rated at $100 \mathrm{MHz}-\mathrm{km}$ and its length is 2 $\mathrm{km}$. Then its maximum frequency is $50 \mathrm{MHz}$. Thus, the maximum operating frequency of fiber is inversely proportional to its length.

\section{A.4. References}

1. Hecht, Jeff, Understanding Fiber Optics, Howard W. Sams and Company, Indianapolis, Indiana, 1987.

2. Nellist, John G., Understanding Telecommunications and Lightwave Systems: An Entry-Level Guide, IEEE Press, New York, 1992, pp. 6, 105-173.

3. Krohn, D. A., Fiber-Optic Sensors: Fundamentals and Applications, 2nd ed., Instrument Society of America, Research Triangle Park, North Carolina, 1992. 


\section{Appendix B}

\section{Glossarry}

$\mu m$ - a micron, one millionth of a meter.

$\mu W-$ a microwatt, one millionth of a watt (a unit of power).

angle of incidence - the angle that a ray of light falling on a surface makes with a perpendicular to the surface at the point of incidence.

angle of reflection - the angle between a reflected ray of light and a perpendicular to the surface at the point of reflection.

attenuation - reduction of signal magnitude, or loss, normally measured in decibels $(\mathrm{dB})$.

avalanche photodiode (APD) - one of two semiconductor devices commonly used to detect light signals and convert them to electrical signals. The APD can detect very faint light. The other device is the PIN diode.

bandwidth - the difference between the high and low frequencies of a transmission band. With regard to light signals, the highest frequency that can be transmitted in analog operation.

bandwidth-length product - the measure of a fiber's ability to transmit high-speed signals, stated in MHz-km. It is influenced by modal dispersion. The bandwidth-length product is used to determine the bandwidth of a particular length of fiber by dividing the length into the bandwidthlength product.

biconic connector - a threaded fiber-optic connector used in high-speed telecommunications. It is designed to bring two fiber ends into physical contact, minimizing loss.

bit - a binary digit, the smallest unit of information in a binary system of notation.

bit rate - the rate, or speed, at which bits (binary digits) are transmitted (e.g., bits per second).

buffer - the protective coating that covers an optical fiber.

CCTV See closed-circuit television. chromatic dispersion - pulse spreading caused by variation in light propagation with wavelength. Units are picoseconds (of dispersion) per kilometer (of fiber length) per nanometer (of source spectral width). Laser sources minimize this effect due to their narrow spectral width.

cladding - the reflective outer layer of an optical fiber that surrounds the light-carrying core. The cladding retains the light in the core and allows the fiber to guide light from one end to the other. The cladding has a lower index of refraction than the core.

closed-circuit television (CCTV) - a television system in which signals are transmitted from the camera to the monitor through electrical wires or an optical fiber, as opposed to broadcast television in which signals are transmitted through the air using antennas.

core - the central part of an optical fiber that carries light. It has a higher index of refraction than the cladding.

critical angle - the angle at which light undergoes total internal reflection.

$d B$ See decibel.

$d B m-\mathbf{a}$ unit of power measured in decibels above (positive) or below (negative) one milliwatt.

decibel $(d B)$ - a logarithmic comparison of two power levels. It can be used to express gain or loss without reference to absolute power levels. Defined as ten times the base-ten logarithm of the ratio of the two power levels.

dispersion - the spreading out of light pulses as they travel along an optical fiber.

dispersion-shifted fiber - specially constructed fiber that shifts the wavelength of minimum chromatic dispersion from $1300 \mathrm{~nm}$ to $1550 \mathrm{~nm}$.

electromagnetic interference (EMI) - noise generated when stray electromagnetic fields induce currents in electrical conductors.

EMI See electromagnetic interference. 
endoscope - a fiber-optic bundle that delivers light and views inside the human body.

fault locator - an instrument used to locate breaks in optical fibers. Fault locators are specialized optical time domain reflectometers.

fiber optics - light transmission through optical fibers for communications, sensing or imaging.

frequency-length product See bandwidth-length product.

fused silica - technical name for glass. It is made by melting silicon dioxide and is commonly used to make optical fiber.

fusion splice - a splice in which two optical fibers are joined using heat to melt the fibers together.

graded-index fiber - optical fiber in which the index of refraction changes gradually with distance from the fiber axis, rather than abruptly at the core-cladding interface.

host computer - in an alarm communications system, the computer that transforms alarms into audible and visible forms usable by the operator. The host computer also accommodates operator control functions and data storage.

index of refraction - same as refractive index. It is a measure of a transparent material's ability to bend light, usually abbreviated " $n$." Index of refraction is the ratio of the speed of light in a vacuum to the speed of light in the material.

intensity-modulated sensor - a fiber-optic sensor that detects changes in light intensity due to motion, vibration, pressure, or other physical effects.

interferometer - an instrument that uses interference of light waves for precise determinations of light wavelength.

interferometry — using interference of light waves to precisely determine light wavelength.

\section{LAN See local area network.}

laser diode - a light-emitting semiconductor device important as a light source in fiber optics. Laser diodes are characterized by their high power and narrow spectral width.

LED See light-emitting diode. light detector - the light receiver in a fiber-optic system that converts optical (light) signals to electrical signals. Two commonly used light detectors are the PIN diode and the avalanche photodiode.

light disturbance sensor - a fiber-optic sensor that detects the disturbance of the light traveling through the fiber. Contrasts with optical continuity sensors, which only detect breaks in the fiber.

light-emitting diode (LED) — a semiconductor device important as a light source in fiber optics. LEDs are relatively inexpensive but have lower power and wider spectral widths than laser diode sources.

light mode - with regard to optical fiber, a stationary vibration pattern of light in the fiber.

light source - the light transmitter in a fiber-optic system that converts electrical signals to optical (light) signals. Two commonly used light sources are the laser diode and the light-emitting photodiode.

local area network (LAN) - a network that transmits data among many nodes in a small area (e.g., a building).

loose tube - a fiber-optic cable construction technique in which the fibers are installed loosely in hollow tubes inside the cable. More than one fiber may be in each tube. This construction technique isolates the fibers from external stresses that the cable might undergo. Contrast with tight buffer construction.

loss margin - extra loss (in $\mathrm{dB}$ ) included in the design of a fiber-optic system to account for age degradation of components, component variation, repairs, and additions (splices and connectors).

MCU See multiplexer control unit.

mechanical splice - a splice in which two optical fibers are joined using a clamp or epoxy.

microbending - tiny bends in optical fiber that allow light to leak out and increase loss.

micron $(\mu m)$ - one millionth of a meter.

modal dispersion - dispersion arising from differences in the times that different modes take to travel through multimode fiber. Also known as multimode dispersion. 
modal interference - interference of the various light modes with each other. Modal interference produces a speckle pattern at the end of a multimode fiber that is being illuminated by a laser.

mode - with regard to optical fiber, a stationary vibration pattern of light in the fiber.

multimode fiber - optical fiber that permits more than one light mode to be propagated. Contrast with single-mode fiber, which allows only one mode to be propagated.

multiplexer - also transponder. In an alarm communications system, the multiplexer is the device that collects alarms from several sensors and relays them to the primary collection device, the multiplexer control unit.

multiplexer control unit (MCU) - in an alarm communications system, the primary alarm collection device. The MCU receives alarms from the multiplexers and interfaces with the host computer.

\section{NA See numerical aperture.}

nanometer $(\mathrm{nm})$ - one billionth $\left(1 \times 10^{-9}\right)$ of a meter.

numerical aperture (NA) - the sine of half the angle over which an optical fiber can accept light for propagation.

optical continuity sensor - a fiber-optic sensor that detects a break in the fiber. Contrast with light disturbance sensors, which detect the disturbance of the light traveling through the fiber.

optical fiber - long strands of transparent material that can guide light. Usually made from glass or plastic.

optical power - light energy per unit time, measured in milliwatts or related units (e.g., dBm). Intuitively, optical power has to do with the brightness of light.

optical power meter - an instrument that measures optical power at a particular wavelength.

optical time domain reflectometer (OTDR) - an instrument that measures transmission characteristics of fiber-optic systems by sending a short pulse of light down a fiber and observing backscattered light. Commonly used for system loss measurements. Locations of fiber breaks, splices, and connectors can also be made with an OTDR.

pan-tilt-zoom (PTZ) - camera controls that affect the field of view. phase - the point in the vibrational or oscillatory period of light.

phase-modulated sensor - a fiber-optic sensor that detects changes in the length of the fiber (actually, changes in the phase of the light) due to motion, vibration, pressure, or other physical effects.

PIN diode - one of two semiconductor devices commonly used to detect light signals and convert them to electrical signals. The PIN diode provides a fast, linear response. The avalanche photodiode is the other device.

power budget - the difference between the power transmitted into an optical fiber and the optical receiver's sensitivity (required receiver power). Power budget is stated in $\mathrm{dB}$.

\section{PTZ See pan-tilt-zoom.}

Rayleigh scattering - scattering of light in a transparent material due to local (microscopic) fluctuations in density and refractive index.

receiver - a light detector that can convert optical (light) signals to electrical signals.

receiver sensitivity - the minimum optical power level required for detection by the receiver. Usually given in $\mathrm{dBm}$.

refraction - the bending of light as it passes between materials of different refractive index.

refractive index - same as index of refraction. It is the measure of a transparent material's ability to bend light, usually abbreviated " $n$." Refractive index is the ratio of the speed of light in a vacuum to the speed of light in the material.

RS-232 - a single-ended electrical serial communications standard that defines the serial link between data terminal equipment (DTE) and data communications equipment (DCE). Can accommodate distances up to 50 feet.

$R S-422$ - an electrical serial communications standard for balanced-line transmission. Can accommodate distances up to 4000 feet.

$R S-485$ - an electrical serial communications standard for balanced-line transmission. RS-485 is an upgrade to RS422 that allows multiple drivers and receivers. Can accommodate distances up to 4000 feet. 
seal - a tamper-indicating device used to ensure the integrity of an item.

single-mode fiber - optical fiber that permits only one light mode to be propagated. Contrast with multimode fiber, which allows more than one mode to be propagated.

SMA connector - a threaded connector commonly used in fiber optics.

speckle pattern - a light interference pattern produced at the end of a multimode fiber that is being illuminated by a laser source.

spectral width - a measure of the wavelength spread of a light source. Specifically, the difference between the wavelengths at which the source's radiant intensity is half its peak intensity.

splice - a permanent junction of two optical fiber ends.

$S T \circledast$ connector - a commonly used fiber-optic connector that incorporates a "twist-lock" feature similar to the coaxial BNC connector.

stabilized light source - a light source that provides a known wavelength and level of optical power. Often used in conjunction with an optical power meter to make loss measurements.

step-index fiber - an optical fiber in which the refractive index changes abruptly at the core-cladding interface. Contrast with graded-index fiber whose refractive index changes gradually from the fiber axis to the core-cladding interface. tight buffer - a fiber-optic cable construction technique in which each fiber in the cable is coated with a buffering material that holds it rigidly in place. This construction technique makes handling of the individual fibers in the cable easier. Contrast with loose tube construction.

total internal reflection - total reflection of light back into a material when it strikes the interface with another transparent material at an angle less than the critical angle. The second material must have a lower index of refraction than the first for this to occur.

transceiver - a device consisting of a transmitter (light source) and a receiver (light detector).

transmitted power - the optical power transmitted into an optical fiber. Usually stated in $\mathrm{dBm}$.

transmitter - a light source that can convert electrical signals to optical (light) signals.

transponder - also multiplexer. In an alarm communications system, the transponder is the device that collects alarms from several sensors and relays them to the primary collection device, the multiplexer control unit.

wavelength division multiplexing (WDM) - multiplexing of signals by transmitting them at different wavelengths through the same fiber.

WDM See wavelength division multiplexing. 


\section{Appendix C}

\section{Standards}

EIA $\quad 440-A-89$

IEEE $812-84$

EIA TIA-458-B-90

EIA $4720000-85$
Fiber-Optic Technology

Standard Definitions of Terms Relating to Fiber Optics

Standard Optical Fiber Material Classes and Preferred Sizes

Generic Specification for Fiber-Optic Cable

EIA TIA-4750000-B-89 Generic Specification of Fiber-Optic Connectors

EIA $5150000-86 \quad$ Generic Specification for Optical Fiber and Cable Splices

EIA TIA-455-57A-90 Optical Fiber End Preparation and Examination

EIA TIA-5730000-93 Generic Specification for FieldPortable Fiber-Optic Tools

EIA TIA-526-92
Standard Test Procedures for Fiber-Optic Systems
EIA CB9-F-87

EIA TIA-455-A-91

EIA TSB19-86

ANSI Z136.2-88

EIA $\quad 515 B 000-87$
Reference Guide for FiberOptic Test Procedures

Standard Test Procedure for Fiber-Optic Fibers, Cables, Transducers, Sensors, Connecting and Terminating Devices, and Other Fiber-Optic Components

Optical Fiber Digital Transmission Systems Considerations for Users and Suppliers

Safe Use of Optical Fiber Communication Systems Utilizing Laser Diode and LED Sources

Sectional Specification for Splice Closures for Pressurized Aerial, Buried, and Underground Fiber-Optic Cables 


\section{Appendix D}

\section{Partial Listing of Fiber-Optics Equipment Suppliers}

Note: Users of this NUREG report should not rely on the completeness of this directory when making purchases of fiber-optics equipment.

\section{D.1 Communications Products}

Allied Telesis

575 East Middlefield Road

Mountain View, CA 94043

(800) 424-4284

(415) $964-2771$

American Fibertek

27 Worlds Fair Drive

Somerset, NJ 08873

(908) 302-0667

Codenoll Technology Corporation

1086 North Broadway

Yonkers, NY 10701

(914) $965-6300$

Fiber Options

80 Orville Drive, Suite 102

Bohemia, NY 11716

(516) $567-8320$

(800) $342-3748$

Force, Incorporated

P. O. Box 2045

(825 Park Street)

Christiansburg, VA 24068

(703) 382-0462

Innovations in Optical Communications, Inc. 9921 Carmel Mountain Road, Suite 242

San Diego, CA 92129

(619) 484-7865

International Fiber Systems Incorporated

(IFS)

34 Delmar Drive

Brookfield, CT 06804

(203) 775-4961
Math Associates Inc. 5500 New Horizons Blyd.

Amityville, NY 11701

(516) 226-8900

Meret Optical Communications, Inc. 1800 Stewart St.

Santa Monica, CA 90404

(310) 828-7496

Optelecom

9300 Gaither Road

Gaithersburg, MD 20877

(301) $840-2121$

Opticomm Corporation

5505 Morehouse Drive, Suite 150

San Diego, CA 92121

(619) 450-0143

\section{D.2 Intrusion Detection Sensors}

Anro Engineering, Inc.

Management Division

1800 Second Street, Suite 878

Sarasota, FL 34236-5992

(813) $957-3080$

Fiber SenSys, Inc.

9640 SW Sunshine Court, \#400

Beaverton, OR 97005

(503) 641-8150

Interactive Technologies, Inc. (ITI)

2266 North Second Street

' North St. Paul, MN 55109

(800) 777-5484

Mason and Hanger National, Inc. 2227 Drake Avenue, Suite 10-D

Huntsville, AL 35805

(205) 881-2728

Perimeter Products

1130 Terra Bella Avenue

Mountain View, CA 94043

(415) $966-8550$ 
Photonic Alarms Products

8130 West 26th Ave.

Hialeah, FL 33016

(800) 237-8277

Remsdaq Limited

Parkway

Deeside Industrial Park

Deeside

Clwyd CH5 2NN

United Kingdom

(0244) 288031

Safeguards Technology Inc.

300 Hudson Street

Hackensack, NJ 07601-6700

(201) 784-0220

Stellar Systems

Santa Clara Technology Park

3511 Leonard Court

Santa Clara, CA 95054

(408) 496-6690

\section{D.3 Seals}

Aquila Technologies Group, Inc. 8401 Washington Place NE Albuquerque, NM 87113 (505) 828-9100

\section{E. J. Brooks Company \\ 164 North 13th Street \\ Newark, NJ 07107 \\ (800) 458-7325}

\section{D.4 Cable}

AT\&T Network Systems National Telemarketing Center 505 North 51 Avenue

Phoenix, AZ 85043

(800) 344-0223

Belden Wire and Cable

P. O. 1980

Richmond, IN 47375

(317) $983-5200$

Mohawk/Cable Design Technologies

9 Mohawk Drive

Leominster, MA 01453

(508) 537-9961

(800) 422-9961
Optical Cable Corporation

P. O. Box 11967

Roanoke, VA 24022-1967

(703) 265-0690

Remee Products Corp.

Fiberoptic Division

186 North Main Street

Florida, NY 10921

(800) 431-3864

(914) 651-4431

The Rochester Corporation

751 Old Brandy Road

Culpeper, VA 22701

(703) 825-2111

Siecor Corporation

489 Siecor Park

Hickory, NC 28603-0489

(704) $327-5000$

Storm Products Co.

Advanced Technology Group

116 Shore Drive

Hinsdale, IL 60521

(708) 323-9121

\section{D.5 Connectors and Termination Tools}

AMP Incorporated

Harrisburg, PA 17105-3608

(800) 522-6752

AT\&T Network Systems National Telemarketing Center

505 North 51 Avenue

Phoenix, AZ 85043

(800) 344-0223

$3 \mathrm{M}$ TelComm Products Division

P. O. Box 2963

Austin, TX 78769-2963

(512) $834-1800$

OFTI

Two Lyberty Way

Westford, MA 01886

(508) 692-6606 
Seiko Instruments USA Inc. Electronic Components Division 2990 West Lomita Blvd. Torrance, CA 90505 (213) $517-7786$

Siecor Corporation 489 Siecor Park

Hickory, NC 28603-0489

(704) $327-5000$

\section{D.6 Splices, Splicing Equipment, and Closure Systems}

Advanced Custom Applications Inc. 6-1 Ilene Court

Belle Mead, NJ 08502

(908) 281-0353

AMP Incorporated

Harrisburg, PA 17105-3608

(800) $522-6752$

AT\&T Network Systems

National Telemarketing Center

505 North 51 Avenue

Phoenix, AZ 85043

(800) 344-0223

GTE Fiber Optic Products

2401 Reach Road

Williamsport, PA 17701

(800) 327-6782

3M TelComm Products Division

P. O. Box 2963

Austin, TX 78769-2963

(512) $834-1800$

PSI Telecommunications Incorporated 3333 North San Fernando Blvd.

Burbank, CA 91504

(818) 843-7944

Reliable Electric/Utility Products

11333 Addison Street

Franklin Park, IL 60131

(312) $455-8010$

Siecor Corporation

489 Siecor Park

Hickory, NC 28603-0489

(704) $327-5000$

\section{D.7 Test and Measurement Equipment}

Ando Electric Co., Ltd.

19-7, Kamata 4-chome

Ota-ku, Tokyo

144 Japan

(03) $733-1151$

Anritsu Corporation

10-27, Minamiazabu 5-chome

Minato-ku, Tokyo 106

Japan

03 (446) 1111

Antel Optronics Inc. 3325B Mainway

Burlington, Ontario

Canada, L7M 1 A6

(416) $335-5507$

AT\&T Network Systems

National Telemarketing Center

505 North 51 Avenue

Phoenix, AZ 85043

(800) 344-0223

EXFO Electro-Optical Engineering Inc. 352 St-Sacrement Ave.

Quebec City, QC, Canada, G1N 3Y2

(418) 683-0211

Fotec Incorporated

The Schrafft Center

Box 246

Boston, MA 02129

(800) 537-8254

(617) $241-7810$

Hewlett -Packard Company

Test and Measurement Group

P. O. Box 58059

MS 5ILSC

Santa Clara, CA 95052-8059

(800) $452-4844$

Laser Precision

109 North Genesee Street

Utica, NY 13502

(315) 797-4449

3M Telecom Systems Group 6801 River Place Blvd.

Austin, TX 78726-9000

(800) 745-7459 
Suppliers

RIFOCS Corporation

474 Constitution Avenue

Camarillo, CA 93012

(805) $389-9800$

Schlumberger Instruments

P. O. Box 7004

829 Middlesex Turnpike

Billerica, MA 01821

(508) 671-9700

(800) 225-5765
Siecor Corporation

489 Siecor Park

Hickory, NC 28603-0489

(704) $327-5000$

Tektronix, Inc.

P. O. Box 1197

Redmond, OR 97756

(800) 833-9200

(503) 923-0333 
Applications of Fiber Optics in Physical Protection

3.

DATE REPORT PUBLISHED

MONTH

March 1994

4. FIN OR GRANT NUMBER L1387

5. AUTHORIS)

\section{TYPE OF REPORT}

Technical

T. H. Buckle

B. PERFORMING ORGANIZATION - NAME AND ADDRESS III NRC, provide Division, Office or Region, U.S. Nuclear Regulatory Commission, and mailing address: If contractor, provide neme and malling eddress)

Sandia National Laboratories

Albuquerque, NM 87185-0781

9. SPONSORING ORGANIZATION - NAME AND ADDAESS iN ARC, type "Same as sbove"; if contractor, pravide NRC Division, Office or Region, U.S. Nuclear Regulatory COmmission, and malling address,l

Division of Fuel Cycle Safety and Safeguards

Office of Nuclear Material Safety and Safeguards

U.S. Nulcear Regulatory Commission

Washington, DC 20555-0001

10. SUPPLEMENTARY NOTES

11. ABSTRACT $(200$ words or lessl

The purpose of this NUREG is to provide technical information useful for the development of fiber-optic communications and intrusion detection subsystems relevant to physical protection. There are major sections on fiber-optic technology and applications. Other topics include fiber-optic system components and systems engineering. This document also contains a glossary, a list of standards and specifications, and a list of fiber-optic vendors.

Fiber Optics

Physical Protection Devices

Data Transmission Systems

Unlimited

14. SECURITY CLASSIFICATION TThis Pogel

Unclassified (This Report)

Unclassified

15. NUMBER OF PAGES

16. PRICE. 